\title{
La adaptación tecnológica como factor de localización industrial. Una revisión de las investigaciones sobre la industria de hilados de algodón en Cataluña (1772-1885)
}

\author{
Technological change as a factor in industrial location. A review of the \\ research into the cotton spinning industry in Catalonia (1772-1885)
}

\author{
OLIVIER RAVEUX Y ALEX SÁNCHEZ \\ CNRS, UMR TELEMME, Aix-en-Provence/Universitat de Barcelona
}

\begin{abstract}
RESUMEN
Este artículo estudia, a partir del caso de la industria de hilados de algodón en Cataluña, el papel desempeñado por el cambio tecnológico en las estrategias empresariales de localización industrial durante la primera fase del proceso de industrialización. Lo que intenta mostrar es que entre tecnología y territorio la relación no es unívoca y que las decisiones empresariales

en materia de localización industrial se ven condicionadas tanto por el sistema tecnológico adoptado como por las características específicas de territorio. A partir de la revisión de la bibliografía más reciente, establece que, en el caso de Cataluña, la implantación territorial de la hilatura del algodón estuvo determinada tanto por la existencia de un tejido industrial previamente establecido, como por el cambio relativo del coste de los factores —energéticos, de transporte y laborales-que fue inducido por la adopción de las innovaciones tecnológicas llegadas en su gran mayoría del extranjero.

PALABRAS CLAVE: Cambio tecnológico, Localización industrial, Hilados de algodón, Cataluña

Código JEL: O3, R3, N6, N63

\section{ABSTRACT}

This article examines, through the analysis of the cotton spinning industry in Catalonia, the role of technological change in corporate strategies for industrial location in the first stage of the industrialisation process. It highlights the lack of consistency in the relation between territory and technology and demonstrates the influence that both the technological system adopted and the territorial features exert on the industrial location decision-making process. Through the review of the most recent bibliography, the article establishes that the location of the cotton spinning industry in Catalonia was determined by the existence of a preestablished industrial framework and by the relative change in the cost that factors such as labour, energy and transport experienced due to the introduction of foreign technology.

KEY WORDS: Technological Change, Industrial Location, Cotton Spinning, Catalonia

JEL Codes: O3, R3, N6, N63
\end{abstract}




\section{Introducción}

$\mathrm{E}$ 1 estudio de la construcción y recomposición de los territorios económicos se ha convertido en los últimos años en uno de los componentes básicos del análisis de los procesos de industrialización. La importancia del territorio en la organización y el desarrollo económico se ha revalorizado a partir sobre todo de los estudios llevados a cabo, inicialmente en Italia, sobre los "distritos industriales". Teniendo como referencia teórica la obra pionera de Alfred Marshall, estos trabajos han constatado las ventajas que se derivaban de la concentración territorial de un gran número de empresas, que, al operar en el mismo sector productivo, establecían entre ellas intensas relaciones de intercambio y coordinación. Ventajas que se concretaban, sobre todo, en forma de economías externas (especialización, mano de obra y costes de transacción), pero también en la generación de una "atmósfera industrial" que favorecía la circulación de conocimientos y la innovación tecnológica ${ }^{1}$.

Uno de los aspectos del estudio del territorio, visto desde la perspectiva económica, que más interés ha suscitado es el de los factores determinantes de la localización industrial. Buena prueba de ello es que ha sido abordado desde diferentes disciplinas científicas - la Nueva Geografía Económica, la Nueva Teoría del Comercio Internacional, la Economía Regional o la Economía Urbana-, ha generado importantes debates académicos y ha llegado a los foros de discusión historiográfica, como se ha podido comprobar en el último Congreso Internacional de Historia Económica celebrado en Helsinki ${ }^{2}$ En estos debates, los argumentos se han ido enriqueciendo y las posiciones - desde aquellas iniciales que otorgaban un papel fundamental a la dotación de recursos, hasta las más recientes que enfatizan sobre todo la importancia del mercado, pasando por las que consideran la herencia manufacturera, entendida sobre todo como "capital social acumulado", un elemento clave en las pautas de localización- evolucionando, pero el interés por el tema no ha decaído y la discusión está lejos de que pueda considerarse concluida ${ }^{3}$.

En nuestro país, la relación entre industria y territorio sólo ha despertado el interés de los historiadores en fechas relativamente recientes ${ }^{4}$. La preocupación principal, como cabía esperar, se ha centrado en el ámbito regional y en la determinación de las causas que expli-

[Fecha de recepción del original, 10 de julio de 2006. Versión definitiva, 31 de marzo de 2009].

1 La actualización, como es bien conocido, del concepto marshalliano de "distrito industrial" arranca con los trabajos de Becattini (1979, 2000, 2002), que han sido el punto de partida de una extensa bibliografía que, en los últimos quince años, ha ampliado los conceptos a la hora de definir las concentraciones industriales en un intento de precisar mejor sus características distintivas. Así, hoy no sólo hablamos de "distritos industriales", sino también de "sistemas productivos locales", "clusters", "territorios innovadores" o "áreas industriales de base endógena". Véase al respecto: Becattini, Bellandi, Dei Ottati y Sforzi (2002), Cooke (2002), Bellandi (2003) y Lescure (2002).

2 Una de las sesiones del Congreso de Helsinki, celebrado en 2006, estuvo dedicada a "The Territorial Dynamics of Industrialization, y fue precedida de dos Coloquios Internacionales, celebrados en Besançon y Neuchatel, que, bajo el título de Les territoires de l'industrie en Europe, 1750-2000, fueron coordinados por Jean Claude Daumas, Pierre Lamard y Laurent Tissot.

3 Una aproximación a la bibliografía internacional sobre el tema, en Parejo (2006) y Tirado, Pons y Paluzie (2006).

4 Existen, no obstante, algunos precedentes importantes, referidos al caso catalán, entre los que destaca el trabajo de Jordi Nadal sobre la industria algodonera catalana publicado en la Història Econòmica de la Catalunya Contemporània. Nadal (1991). Estudios históricos sobre distritos industriales en España, destacan los de Miranda (2004, 2005) y Raveux (2005). 
carían las desigualdades regionales en la localización industrial ${ }^{5}$. También en nuestro caso, a la hora de establecer los factores claves de la implantación territorial de la industria española, se ha puesto el acento en aspectos como el acceso a los recursos naturales, la disponibilidad de capital humano o el tamaño del mercado. Como han señalado recientemente Tirado, Pons y Paluzie, la especialización industrial regional en España no "estuvo sólo relacionada con la dotación relativa de recursos (...), debería asociarse también a la existencia de economías de aglomeración ligadas al tamaño del mercado" ${ }^{6}$.

Estos factores, sin embargo, se han considerado principalmente en función de la concentración regional de la industrialización española y han sido analizados, en consecuencia, desde una perspectiva básicamente interregional. Pero cabe preguntarse si a nivel intrarregional estos factores servirían igualmente para explicar la localización territorial de la industria o si, en este caso, no habría que considerar también en el análisis otros factores e incluso otros marcos territoriales.

Estas dudas son, en gran medida, las que han llevado a Antonio Parejo a considerar que la dimensión urbana — la ciudad como ámbito natural de localización industrial— resulta probablemente mucho más adecuada que la regional a la hora de captar la verdadera relación entre industria y territorio en los procesos de industrialización ${ }^{7}$. Y estas dudas son también las que nos han llevado a nosotros a plantearnos si entre en los factores determinantes de la localización industrial no habríamos de prestar mayor atención a la tecnología.

El objetivo de este trabajo es considerar el papel del cambio tecnológico en la localización industrial durante la primera fase de la industrialización. Lo que intenta es mostrar que entre tecnología y territorio hay mucha más relación de la que normalmente se ha considerado y que esta relación no es unívoca, es decir que no circula sólo en una dirección $^{8}$. Es evidente que la tecnología es un factor condicionante de las estrategias empresariales, pero también lo es el territorio. La opción tecnológica que haga un empresario puede determinar el emplazamiento territorial de su empresa, debido a los requerimientos energéticos o laborales que se deriven de las características específicas de una innovación técnica. Pero también el territorio puede condicionar la elección tecnológica, al forzar al empresario a adoptar sólo aquellas tecnologías que mejor se adapten a las características geográficas, comerciales o productivas de una determinada zona. En el primer caso, cuando el cambio en el sistema tecnológico tiene repercusiones territoriales, la dotación de recursos desempeña un papel muy importante en las decisiones empresariales. En el segundo, cuando es el territorio el que condiciona la adopción del sistema tecnológico, éstas están sobre todo influenciadas por las características del mercado y las especializaciones productivas previamente establecidas.

Evidentemente, en este artículo no pretendemos abordar el tema en su globalidad, sino hacerlo a partir de un ejemplo concreto, como es el de la industria catalana de hilados

Este interés por la dimensión regional de la industrialización española tiene su fundamento historiográfico en las obras de Pollard $(1981,1994)$ y Hudson $(1989)$, y se ha concretado sobre todo en: Nadal y Carreras (1990), Germán, Llopis, Maluquer de Motes y Zapata (2001), Domínguez (2002), Paluzie, Pons y Tirado (2002), Tirado, Pons y Paluzie (2003), Rosés (2003) y Tirado, Pons y Paluzie (2006).

6 Tirado, Pons y Paluzie (2006), p. 60.

Parejo (2006).

8 La bibliografía específica sobre esta cuestión no es muy abundante. A nivel teórico destaca la obra de Feldman (1994) y a nivel histórico se pueden consultar Maluquer de Motes (2000) para el tema de los "milieux innovateurs" y el reciente número de Histoire, Economie, Société (2007/2) dedicado a "Les logiques spatiales de l' innovation (XIXe-XXe siècles)". 
de algodón durante la primera industrialización ${ }^{9}$. Esta industria, que fue la primera que se mecanizó en Cataluña, iniciando con ello el proceso de modernización industrial en España, resulta especialmente adecuada para estudiar el tema por diversas razones. En primer lugar, por la diversidad de sus localizaciones espaciales en los siglos XVIII y XIX, que contempla desde la manufactura dispersa en medios rurales y urbanos hasta las colonias y los distritos industriales. En segundo lugar, por la presencia de tres sistemas energéticos -animal, hidráulico y vapor- que han contribuido activamente al establecimiento y a la evolución de las pautas de localización territorial. Y, en tercer lugar, por la importancia que las innovaciones técnicas han tenido en esta industria, tanto por su intensidad como por su precocidad ${ }^{10}$. Por ello, pensamos que esta aproximación monográfica sectorial, cuyo objetivo principal es plantear una nueva reflexión sobre el tema a partir sobre todo de la revisión de la literatura más reciente, nos puede permitir valorar la importancia, y la ambivalencia, de las herencias industriales y entender mejor las relaciones que se han establecido a largo plazo entre innovación tecnológica, transformación del territorio y dinamismo empresarial.

\section{Sistemas tecnológicos e implantación territorial en los inicios de la industrialización catalana (1772-1832)}

En Cataluña, la hilatura del algodón fue una actividad de desarrollo tardío. Durante la mayor parte del siglo XVIII, la manufactura de indianas se expansionó sobre la base de integrar en una misma unidad productiva las fases del tejido y del estampado de las telas de algodón, pero no la del hilado. La materia prima utilizada fue el algodón ya hilado procedente sobre todo de la isla de Malta. Esta situación provocó que la difusión de la hilatura en el Principado, que se produjo durante el último cuarto de siglo XVIII, coincidiera prácticamente con el proceso de cambio tecnológico que estaba transformando la industria algodonera en Europa. De ahí, que las innovaciones técnicas - las nuevas máquinas de cardar y de hilar - se adoptaran con relativa rapidez — dos décadas - y contribuyeran no sólo a impulsar el desarrollo de la hilatura, tanto la manual como la mecánica, sino a configurar también su implantación territorial, dibujando ya el mapa básico de la Cataluña algodonera del siglo XIX.

9 El trabajo abarca cronológicamente el periodo 1772-1885. Es una visión, por tanto, de largo plazo que arranca con la creación de la Real Compañía de Hilados de Algodón, que fue la primera en organizar una amplia red de trabajo doméstico dedicada a la hilatura del algodón, y concluye a mediados de los años ochenta del siglo XIX, que es el momento en que se consolidan los cambios que se han ido produciendo desde los años cincuenta en la distribución regional de la industria del hilado y que ya no se verán alterados hasta la irrupción de un nuevo sistema energético, a principios del siglo xx, basado en la electricidad, que alterará sustancialmente las pautas de localización y que por su importancia y complejidad requeriría un trabajo específico.

10 No hemos incluido en este trabajo la industria del tejido para mantener la coherencia cronológica del proceso (el tejeduría se mecanizó más lentamente), si bien durante la segunda mitad del siglo XIX, a medida que avanza el proceso de integración vertical de las empresas, las diferencias desaparecen en gran medida. No obstante, aunque sospechamos que buena parte de nuestros argumentos serían aplicables a la industria del tejido, son muchas aún las incógnitas que tenemos sobre este subsector para poder afirmarlo con rotundidad. 


\subsection{Los inicios de la hilatura manual, $\mathbf{1 7 7 2 - 1 7 8 5}$}

Hasta principios de la década de 1770, la hilatura del algodón fue una actividad marginal. Se hilaba algodón, por supuesto, pero en pequeñas cantidades y como actividad complementaria y estacional de las fábricas de indianas, en función de las necesidades de las empresas y de las fluctuaciones coyunturales del mercado del algodón hilado de Barcelona. Esta actividad se limitaba a las grandes fábricas y estaba organizada básicamente en forma de industria domiciliaria. Aunque estas empresas disponían de tornos para hilar y ocasionalmente hilaban en el propio establecimiento, lo habitual era repartir algodón entre hiladoras particulares que trabajaban a tiempo parcial en sus domicilios tanto en la propia ciudad como, sobre todo, en pueblos situados en comarcas próximas a la capital ${ }^{11}$.

Esta situación empezó a cambiar en los años setenta y en ello tuvo mucho que ver la Real Compañía de Hilados de Algodón de América. Esta sociedad, constituida en 1772 y que agrupaba a los fabricantes de indianas de Barcelona, surgió con el doble objetivo de acabar con el monopolio maltés en la provisión del algodón hilado y de fomentar en Cataluña el hilado del algodón en rama procedente de las colonias americanas. Empresa atípica, resultado de la confluencia de intereses de la Corona y de los grandes fabricantes de indianas, fue pionera tanto en el fomento del hilado del algodón en Cataluña como en la introducción de las primeras innovaciones técnicas y organizativas ${ }^{12}$. Durante su primera etapa, entre 1772 y 1775, estableció las bases de un modelo de hilatura rural y dispersa aprovechando las redes establecidas en los años sesenta por algunas fábricas de indianas en zonas relativamente próximas a Barcelona, donde existía una tradicional industria lanera. Pero fue a partir de su reconstitución en 1783, coincidiendo con el inicio de un gran ciclo expansivo de la manufactura de indianas y lienzos pintados, que estuvo estrechamente ligado al crecimiento del comercio colonial, cuando la Compañía contribuyó de forma sustancial a impulsar la hilatura en Cataluña ${ }^{13}$.

Efectivamente, entre 1783 y 1789, la Compañía de Hilados, una vez asegurado el suministro de materia prima procedente de las colonias, pudo organizar una amplia red de trabajo domiciliario que permitió difundir la hilatura del algodón por una parte importante del territorio catalán. Para ello, siguió una doble pauta de localización. En primer lugar, aquellas áreas donde existía ya una cierta tradición en el trabajo de hilar tanto la lana como el algodón, pero sin que ésta fuera una especialización muy arraigada y bien organizada por empresarios locales que pudiera plantear serios problemas de competencia. Era el caso de comarcas de la Cataluña central y prelitoral bien conectadas comercialmente con Barcelona. En segundo lugar, zonas agrícolas donde no había arraigado la industria textil o ésta era muy marginal, y que, por tanto, la Compañía podía "colonizar" introduciendo una actividad totalmente

11 Sobre estas primeras prácticas de hilatura: Delgado (1990), pp. 166-168; Thomson (1994), pp. 283 y 285 y García Balañà (2004), pp. 57-59. La escasa relevancia de esta actividad la certificaba la Junta de Comercio de Barcelona cuando afirmaba, en 1815, que: "Hace medio siglo ni aún para torcidos se hilaba aquí ni en ninguna parte del Reyno y era Malta la que se aprovechaba con sus introducciones de una desidia tan perjudicial como ruborosa". Citado en Sánchez (2000b), p. 175.

12 Sobre la Compañía ver Sánchez (1987); Thomson (1994); Okuno (1999) y García Balañà (2004).

13 El descubrimiento del archivo de la Compañía y los estudios que, a partir de esta nueva documentación, han realizado Okuno y García Balañà en los últimos años, es lo que ha permitido revisar y revalorizar el papel desempeñado por la misma en la primera expansión de la hilatura en Cataluña, que, en parte, había sido subestimado en los primeros trabajos de Sánchez y Thomson, citados en la nota anterior. La excepcional coyuntura 1783-1796 y su relación con la expansión de la hilatura puede verse en Sánchez (2000a). 
nueva. En esta situación se encontraban las comarcas del oeste y del sur del Principado próximas a Lleida y Tarragona. Evidentemente, las características organizativas de la hilatura fueron distintas en cada una de estas áreas. En las comarcas laneras, la Compañía dependía de las formas de organización del trabajo previamente establecidas y, en consecuencia, se veía obligada a contratar pelaires y tejedores que controlaban las redes de trabajo domiciliario y actuaban como pequeños empresarios locales. Ello tenía la ventaja de facilitar una rápida implantación, ya que se podía aprovechar la organización y los conocimientos técnicos y comerciales previamente existentes, pero también presentaba el inconveniente del elevado precio y volatilidad de la mano de obra, derivados en gran medida de la competencia que ejercían empresarios privados. Esta competencia explica que la Compañía evitara justamente aquellas poblaciones donde la hilatura del algodón empezaba a tener una cierta importancia, como Berga, Capellades, Igualada u Olot, o donde la industria lanera tenía una gran fuerza social y económica, como Terrassa, Monistrol o Esparraguera.

Por el contrario, en las comarcas sin tradición lanera, la hilatura se organizó en forma de "factorías" controladas directamente por la Compañía. En estos establecimientos, dirigidos por un "factor" o representante a sueldo de la empresa, se recibía el algodón en rama de Barcelona, se procedía a su cardado en el propio almacén, después era distribuido entre las hiladoras que trabajaban en sus casas a destajo y, finalmente, se recogía para ser remitido a la capital. Era, por tanto, una forma de organización que combinaba el trabajo concentrado - en el cardadoy el trabajo disperso — en la hilatura-, y que resultaba necesaria ya que, dada la falta de experiencia en el trabajo de cardar e hilar que había en estas comarcas, facilitaba el control de la mano de obra y favorecía su aprendizaje. Pero tenía el problema de su elevado coste, que no era tanto consecuencia del precio de la mano de obra, en general más barata que en las otras áreas, como de los gastos derivados del proceso de formación de cardadores e hilanderas, del mantenimiento de las instalaciones y del transporte de la materia prima y del producto acabado desde y hacia Barcelona. Si a ello añadimos la deficiente calidad del hilo obtenido tendremos las claves que explican que en 1787 la Compañía, después de evaluar que el coste de las "factorías" en sus tres primeros años de funcionamiento había ascendido a 12.979 libras, decidiera abandonar este sistema y con él la hilatura en la mayoría de estas comarcas ${ }^{14}$.

De hecho, la Compañía no sólo dejó de hilar en estas comarcas, sino que redujo también sustancialmente su actividad en el resto de zonas a partir de ese momento. La causa principal fue la irrupción de la competencia privada que la Compañía no podía ni quería enfrentar ${ }^{15}$. En la segunda mitad de los años ochenta y durante toda la década siguiente, la hilatura se convirtió en una oportunidad de negocio que atrajo cada vez a mayor número de empresarios, muchos de ellos procedentes de las filas de los pelaires y tejedores que habían participado en la creación de las redes de trabajo doméstico en los años anteriores ${ }^{16}$. A partir de ese momento, la hilatura dejó de ser una actividad organizada en gran medida por una empresa colectiva dirigida desde Barcelona para convertirse en una industria popular practicada por numerosas pequeñas empresas particulares establecidas en una amplia franja del territorio.

14 Biblioteca de Cataluña (BC en adelante), Fondo Gónima, Caja 47/5. Informe sin fecha (aunque por su contenido se deduce que es de 1787).

15 También contribuyó a este paulatino abandono de la actividad hiladora en régimen de industria rural el hecho de que la Compañía, como veremos después, había instalado en 1787 en Barcelona el primer taller de hilados con máquinas jenny que funcionó en Cataluña.

16 En 1787, la Compañía se refería ya al "crecido número de traficantes y Fábricas que repetidamente van erigiéndose en el interior de nuestra Provincia". BC. Fondo Gónima, L. 12, Llibre de resolucions (1783-1794), sesión del 8 de octubre de 1787. 
Aunque la Compañía de Hilados no llegó a monopolizar la producción de hilados en Cataluña durante estos años, sí contribuyó de forma decisiva a su difusión en el territorio ${ }^{17}$. En 1784, la Compañía afirmaba que: "Es ya de mucha consideración la cantidad de algodones en rama que se hilan en Cataluña, tanto por cuenta de dicha Sociedad, como por la de otros particulares, llegando al número de 2.000 arrobas en cada mes, en cuya operación se hallan empleadas más de 6.000 mugeres y unos 750 hombres en clase de cardadores ${ }^{\prime 18}$. Las cifras quizá sean un tanto exageradas, pero lo importante es que esa mano de obra se extendía ampliamente por la geografía catalana. Como puede verse en el cuadro 1, en 1784-1785, justo en el momento en que aparecía la jenny en Barcelona, la Compañía hilaba algodón en 102 pueblos situados en 18 comarcas catalanas. El grueso de la producción se concentraba, no obstante, en seis de ellas, tres situadas en zonas de larga tradición lanera - el Vallès Oriental, Osona y l'Anoia, que representaban el 57,1\% del total de algodón hilado por la Compañía en esos dos años-y las otras tres en áreas sin industria previamente establecida - la Segarra, el Solsonés y la Conca de Barberà, que reunían el $26,6 \%{ }^{19}$. Esta primera geografía del hilado en Cataluña se completaba lógicamente con algunas poblaciones donde la presencia ya de una notable industria del hilado del algodón había actuado como barrera de entrada para la Compañía, de las cuáles las únicas importantes que podían ampliar la nómina comarcal eran Berga (el Berguedà) y Olot (la Garrotxa) ${ }^{20}$.

CUADRO 1

LA HILATURA DE ALGODÓN EN CATALUÑA EN 1784-1785 (ALGODÓN HILADO POR LA COMPAÑÍA DE HILADOS DE ALGODÓN DE BARCELONA)

\begin{tabular}{llcc}
\hline \multicolumn{1}{c}{ Comarcas } & \multicolumn{1}{c}{ Capitales } & Algodón hilado $\mathbf{( k g )}$ & $\%$ \\
\hline 1. Vallès Oriental & Granollers & $36.143,32$ & 34,53 \\
2. Segarra & Cervera & $16.013,92$ & 15,25 \\
3. Osona & Vic & $12.984,19$ & 12,4 \\
4. Anoia & Igualada & $10.741,53$ & 10,26 \\
5. Solsonès & Solsona & $6.607,22$ & 6,33 \\
6. Conca de Barberà & Montblanc & $5.413,4$ & 5,18 \\
7. Camp Tarragona & Tarragona & 3.588 & 3,43 \\
8. Ripollès & Ripio & $2.206,46$ & 2,11 \\
9. Vallès Occidental & Sabadell & $2.021,76$ & 1,93 \\
10. Alt Penedès & Vilafranca del Penedès & $1.788,38$ & 1,71 \\
11. La Selva & Santa Coloma de Farners & $1.669,82$ & 1,6 \\
12. Baix Llobregat & Sant Feliu de Llobregat & $1.666,08$ & 1,59 \\
13. Segrià & Lleida & $1.067,04$ & 1,02 \\
14. Urgell & Urgell & 809,95 & 0,77 \\
15. Maresme & Mataró & 797,47 & 0,76 \\
16. Bages & Manresa & 720,09 & 0,69 \\
17. Noguera & Balaguer & 246,83 & 0,33 \\
18. Barcelonés & Barcelona & 118,56 & 0,11 \\
\hline
\end{tabular}

Fuentes: Okuno (1999), p. 70-72 y García Balañà (2004), p. 72-77.

17 Entre 1783 y 1789, la Compañía hiló casi 170 toneladas de algodón, una cantidad ciertamente considerable. Okuno (1999), pp. 67 y 67.

18 BC. Fondo Gónima, Caja 44/5, Representación al Rey de julio de 1785 con motivo de la formación de la Real Compañía de Filipinas.

19 La distribución comarcal, en Okuno (1999), pp. 70-72 y García Balañà (2004), pp. 72-77.

20 Para los casos de Berga y Olot, Solà $(1995,2002)$. 
Este primer mapa de la hilatura en Cataluña experimentó algunas modificaciones relevantes en las dos décadas siguientes. Lo más significativo fue que la hilatura se concentró en aquellas zonas donde había existido un previo tejido industrial y desapareció prácticamente de las de nueva "colonización". La pauta de localización que se consolidó fue justamente la primera de las que practicó la Compañía, confirmando la afirmación de Jaume Torras de que la difusión de la actividad algodonera en Cataluña se hizo "en función de la previa implantación de industrias rurales, que preparaba la oferta de trabajo y la organizaba"21. En 1804, según un documento de la Junta de Comercio en el que se relacionan las poblaciones a las que se había enviado desde Barcelona algodón en rama para su hilado en los dos primeros meses del año, la hilatura abarcaba 64 pueblos pertenecientes a una quincena de comarcas $^{22}$. La extensión geográfica era ahora menor, pero lo más importante es que casi el $90 \%$ de la producción se concentraba, como puede verse en el cuadro 2, en seis comarcas que, en su gran mayoría, contaban con una consolidada tradición manufacturera, y que eran por orden de importancia:

CUADRO 2

LA HILATURA DE ALGODÓN EN CATALUÑA EN 1804 (ALGODÓN DISTRIBUIDO POR COMARCAS DESDE BARCELONA DURANTE LOS MESES DE ENERO Y FEBRERO DE 1804 PARA SU HILADO)

\begin{tabular}{llcc}
\hline \multicolumn{1}{c}{ Comarcas } & \multicolumn{1}{c}{ Capitales } & Algodón en rama $\mathbf{( k g )}$ & $\%$ \\
\hline 1. Anoia & Igualada & 56.550 & 21,9 \\
2. Osona & Vic & 54.844 & 21,2 \\
3. Berguedà & Berga & 44.062 & 17,2 \\
4. Bages & Manresa & 29.325 & 11,5 \\
5. Garrotxa & Olot & 17.875 & 6,9 \\
6. Vallès Occidental & Sabadell & 10.460 & 4,2 \\
7. Maresme & Mataró & 8.463 & 3,3 \\
8. Segarra & Cervera & 6.383 & 2,5 \\
9. Baix Camp & Reus & 5.510 & 2,2 \\
10. Alt Camp & Valls & 5.085 & 1,9 \\
11. Conca de Barberà & Montblanc & 4.819 & 1,8 \\
12. Baix Llobregat & Sant Feliu de Llobregat & 3.072 & 1,3 \\
13. Ripollès & Ripio & 2.559 & 1,1 \\
14. Moianès & Moià & 2.360 & 0,9 \\
15. Solsonès & Solsona & 1.437 & 0,5 \\
16. Alt Penedès & Vilafranca del Penedès & 1.085 & 0,4 \\
17. Pla de l'Estany & Banyotes & 1.085 & 0,4 \\
18. Gironès & Girona & 862 & 0,3 \\
19. Vallès Oriental & Granollers & 853 & 0,3 \\
20. Cerdanya & Puigcerdà & 437 & 0,1 \\
21. Alt Empordà & Figueres & 376 & 0,1 \\
22. Pallars Jussà & Tremp & 155 & 0 \\
23. Barcelonès & Barcelona & 132 & 0 \\
24. Baix Penedès & El Vendrell & 8 & 0 \\
\hline
\end{tabular}

Fuente: Solà (2004a), pp. 267-268.

21 Torras (1994), p. 31.
22 Documento reproducido en el apéndice del libro de Angels Solà (2004a), pp. 267-268. 
l'Anoia, Osona, el Berguedà, el Bages, la Garrotxa y el Vallès Occidental. Si a ellas le sumamos Barcelona, que no está incluida en esta relación pero que ya en estos años contaba con una importante industria de hilados, tendremos el mapa básico de la hilatura catalana del primer tercio del siglo XIX, en el que destacan sobre todo las comarcas de la Catalunya central y prelitoral que envuelven las cuencas de los ríos Llobregat y Ter y la capital ${ }^{23}$. Ahora bien, en 1804 el mapa de la hilatura no era sólo el de la hilatura manual, era también el de la hilatura hecha con máquinas, en unos casos movidas a mano y en otros mediante animales o agua. Desde hacía unos años, el cambio técnico estaba contribuyendo ya a configurar la distribución territorial de la nueva industria.

\subsection{La hilatura mecánica: innovaciones técnicas y primera localización territorial (1785-1832)}

Las máquinas que revolucionaron la industria algodonera y que supusieron el inicio del proceso de industrialización llegaron a Cataluña entre 1785 y 1806. Veinte años llevó completar el ciclo tecnológico que llevaba de la jenny a la mule-jenny. Un periodo de tiempo que, en gran medida, coincidió con el proceso de implantación de la hilatura, por lo que bien puede decirse que, en Cataluña, las máquinas acompañaron y estimularon este proceso ${ }^{24}$.

Con la aparición de las máquinas de hilar clásicas se configuraron, de hecho, tres sistemas tecnológicos distintos: el de la jenny, el de la water y el de la mule. Cada uno de ellos suponía algo más que la propia máquina y sus específicas características técnicas — procesos preparatorios, tipo de hilo y productividad-, implicaba también las condiciones económicas - coste e instalación-, sociales —organización del trabajo- y energéticas -tipo de motor para moverlas- que hacían posible y rentable su utilización. En Cataluña, estos tres sistemas se configuraron en los años del cambio de siglo y perduraron hasta la década de 1840. No lo hicieron, además, compitiendo entre sí. De hecho, más que sistemas alternativos fueron complementarios. No se produjo de forma generalizada una sustitución de máquinas en función de su mayor productividad. La jenny no fue desplazada por la water y ésta a su vez por la mule. Lo que se dio fue complementariedad entre algunas de ellas y coexistencia de todas durante un largo periodo de tiempo. Y en ello, tanto como las propias características técnicas de cada máquina, tuvo mucho que ver el territorio. En cada zona, como muy bien ha señalado Llorenç Ferrer, se adoptaron aquellos sistemas tecnológicos que mejor se adaptaban a la

23 La importancia de Barcelona en los inicios de la hilatura en Cataluña era señalada ya por la Compañía de Hilados en 1783, cuando afirmaba que: "La hilanza de los Algodones a que dieron principio algunos particulares Dueños de Fábricas, y continuó después la expresada Compañia, tuvo su primer origen y establecimiento dentro de esta vuestra ciudad: en ella se buscó el modo más fácil de despepitar el algodón y el mejor método de cardarlo; en ella se hicieron los primeros tornos y se adiestraron las primeras manos para hilarlo". BC. Fondo Gónima, Caja 44/3, Representación al Rey de 31 de mayo de 1783. En los primeros años del siglo XIX, la capital seguía desempeñando un papel clave en el desarrollo del sector, tanto por el número de empresas radicadas en la ciudad, como por su contribución al cambio tecnológico. En ella se dieron a conocer por primera vez las nuevas máquinas de hilar y hacia 1807 concentraba el mayor número de ellas de toda Cataluña (había, como mínimo, 349 jennies, 43 water-frames y 14 mule-jennies).

24 Los trabajos que ofrecen una visión general sobre este tema son: Sánchez (2000a, 2000b), Solà (2002, 2004a) y Thomson (2003a, 2003b, 2005). Sobre la transferencia de tecnología en Europa y el papel desempeñado por los técnicos ingleses: Berg y Bruland (1998), Chassagne (1991) y Harris (1998). 
estructura productiva ${ }^{25}$. Pero también se podría decir que cada sistema tecnológico tendió a ubicarse allí donde mejor se podía desarrollar su potencial productivo.

En el caso de la jenny y de sus versiones mejoradas, incluida la bergadana, lo primero que hay que tener presente es que se trataba de máquinas relativamente sencillas, movidas por fuerza humana, que tenían un precio moderado - entre 25 y 50 libras - y producían un hilo de trama en general grueso que se utilizaba para fabricar los tejidos crudos llamados empesas. Por ello, resultaban adecuadas para ser empleadas en domicilios y no tenían porqué alterar las pautas organizativas de la hilatura tradicional. De hecho, reforzaban el carácter de industria rural y dispersa, intensiva en trabajo, que había tenido la hilatura manual hasta ese momento. Se trataba, por tanto, de máquinas que podían difundirse extensamente por el territorio porque se adaptaban fácilmente al vigente sistema del putting-out.

Pero en la práctica, la tecnología de la jenny implicó cambios significativos en las formas de organización del trabajo. En especial, favoreció su concentración en talleres y pequeñas fábricas. Esta fue, como ha señalado Albert García Balañà, una opción organizativa de los empresarios con mayores recursos que buscaban maximizar sus rentas monopolizando la nueva tecnología y centralizando el trabajo para aumentar su productividad ${ }^{26}$. Las jenny factories se pueden encontrar en casi todos los países europeos, pero sólo fueron significativas en las primeras fases de implantación de la nueva tecnología, cuando aún no se había producido la "difusión social" de la innovación y no se conocían los problemas que implicaba controlar una mano de obra, mayoritariamente femenina, acostumbrada a unas prácticas laborales muy alejadas de la regularidad y disciplina que implicaba el trabajo fabril ${ }^{27}$. Por ello, la tendencia que se impuso posteriormente fue la externalización del trabajo y la subcontratación, que dieron lugar a la formación de pequeños talleres que operaban con pocas máquinas y sobre la base del trabajo familiar ${ }^{28}$.

Este proceso se dio también en Cataluña. La primera utilización de la jenny fue justamente en el taller centralizado que la Compañía de Hilados montó en Barcelona en 1787 y que tres años después tenía 21 máquinas y empleaba unos 70 trabajadores. Pero no fue el único. Desde esa fecha se constituyeron diversas sociedades, tanto en Barcelona como en otras poblaciones del Principado, que reproducían el mismo modelo organizativo, aunque la mayoría tuvieron una vida corta ${ }^{29}$. La flexibilidad y bajo coste de la máquina, unida a los problemas de control y disciplina de la mano de obra y a la existencia de numerosos artesanos con escaso capital pero con conocimientos en el sector y dispuestos a aprovechar nuevas oportunidades de negocio, explican que en Cataluña se impusieran el pequeño taller y la industria a domicilio como característicos del sistema tecnológico de la jenny ${ }^{30}$. No obstante, hasta 1808 siguieron existiendo empresas de cierta envergadura. En 1802, por ejemplo, de los 12 empresarios del

Ferrer (2004), p. 341.

García Balañà (2004), pp. 155-161.

Berg (1987), pp. 261-262; Berg, Hudson y Sonenscher (1983), pp. 11-13 y Reddy (1984), pp. 51-57.

Lazonick (1990), pp. 80 y ss.

29 Fue el caso también del más importante fabricante de indianas de Cataluña, Erasmo de Gónima, que entre 1796 y 1802 montó una fábrica de hilados en Barcelona equipada con 22 jennies y 3 máquinas de cardar por cilindros. Sobre estas primeras jennies factories en Cataluña, véase García Balañà (2004), en especial el capítulo 3.

30 En Barcelona, en los primeros años del siglo XIX, muchos de estos talleres ocupaban bajos y tiendas de casas, pero también pisos, como señalaba un fabricante de 1’Espluga de Francolí (la Conca de Barberà) haciéndose eco de las informaciones que le llegaban de Barcelona: “...del que me diu que en eixa se treballa per los pisos, no y tinch res que dir que ja ho crech". Citado en García Balañà (2004), p. 209. 
Cuerpo de Fabricantes de Tejidos e Hilados de Barcelona que declaraban tener máquinas de hilar, la mitad poseían más de 20 unidades $^{31}$. Después de la guerra el modelo organizativo basado en el pequeño taller se impuso claramente entre los fabricantes que hilaban con jennies y bergadanas ${ }^{32}$.

Dada su versatilidad y bajo coste la jenny se difundió ampliamente por el territorio catalán, localizándose sobre todo en aquellas poblaciones y comarcas donde había arraigado la hilatura manual. El carácter disperso de esta hilatura hace muy difícil determinar con precisión en qué zonas se utilizaron este tipo de máquinas. Sólo cuando se empleaban en talleres han dejado rastro documental. Por eso, sabemos que entre 1802 y 1808 su geografía abarcaba comarcas como el Barcelonés, el Baix Camp, la Garrotxa, el Bages, Osona, l'Anoia, el Berguedà o la Conca de Barberà, pero que tendía a concentrarse sobre todo en un conjunto de ciudades o pueblos de larga tradición textil, como Barcelona, Reus, Olot, Vic, Berga, Sallent, Cardona, Manresa y, probablemente, Igualada ${ }^{33}$. Una geografía que cambió muy poco en el primer tercio del siglo XIX. En 1841, justo cuando este tipo de tecnología iniciaba su decadencia, las poblaciones y comarcas que concentraban el mayor número de bergadanas eran prácticamente las mismas que a principios de siglo. Lo único que había cambiado era la jerarquía. Ahora la lista la encabezaban Igualada y Berga y la cerraban Barcelona y Reus ${ }^{34}$.

Al ser muy poco intensiva en capital y energía y mucho en trabajo, la clave de la implantación de este tipo de tecnología hay que buscarla en la dotación de capital humano y en el tamaño del mercado. Lo determinante fue la disponibilidad de mano de obra cualificada y de empresarios - artesanos y comerciantes - con capacidad y conocimientos para organizar el trabajo y las redes de comercialización del producto. Y estos factores sólo se encontraban en aquellas zonas donde se había dado previamente un proceso de especialización industrial, como eran justamente las comarcas de la Cataluña central con el trabajo de lana, o que estaban próximas a los grandes mercados de consumo, que en este periodo eran sobre todo Barcelona, Reus y Olot.

Por supuesto, estos factores actuaron también en la implantación territorial de los otros dos sistemas tecnológicos, pero no fueron los únicos. En estos casos estuvieron acompañados por otros que en gran medida resultaron decisivos, ya que tenían que ver con sus propias características técnicas y sus requerimientos energéticos.

La water-frame era una máquina diseñada como parte integrante de un sistema de hilar continuo que incluía también máquinas preparatorias y que debía ser movido por medio de energía hidráulica. Se trataba, por tanto, de una tecnología muy intensiva en capital, no sólo por el coste de la maquinaria -la water costaba unas 500 libras a principios de siglo y otras tantas la carda de cilindros-, sino también por el de las instalaciones que se necesitaban para albergarlas. Estas máquinas difícilmente se podían instalar en domicilios particulares o en pequeños talleres; requerían establecimientos de mayores dimensiones dotados de un salto

31 La fábrica de Joan Serra, por ejemplo, tenía 48 máquinas, la de Manuel Torruella 46 o la de Joan Cabirol 26. Sánchez (1989), p. 87.

32 En 1829, de los 40 que lo hacían con este tipo de máquinas en Barcelona, el 75\% tenían menos de 10 unidades. Padrón de Fabricantes de Barcelona de 1829, publicado en Graell (1911), pp. 422-423.

33 En algunas de estas poblaciones, como Berga, Cardona o Sallent, la hilatura con jennies se combinaba con el cardado hecho con máquinas de cilindros movidas por agua, pero normalmente esta fase preparatoria se realizaba en talleres independientes de los que hilaban. Por el contrario, cuando el cardado se hacía a mano o mediante caballerías lo habitual era que las dos funciones estuvieran integradas en el mismo taller o fábrica. Ferrer (2004) y Solà (1995).

34 La estadística de 1841 en Ferrer (2004). 
de agua ${ }^{35}$. A cambio, esta tecnología era menos intensiva en trabajo. Como ha puesto de manifiesto Albert García Balañà, exigía menos trabajo diestro y menos fuerza. El trabajador no era tan decisivo en este sistema que en los otros y, por tanto, más fácil de reemplazar. Incluso se le remuneraba diferente, a jornal, cuando lo normal era a destajo. De ahí, que fuera un trabajo muy feminizado ${ }^{36}$. Otra característica distintiva de esta máquina es que producía sólo hilo de urdimbre y ello condicionaba su uso, ya que la convertía en una tecnología complementaria de las otras que producían hilo de trama, en especial de la jenny. Por ello, era frecuente que las primeras fábricas de hilados mecánicas también tuvieran este tipo de máquinas más simples, sobre todo si la empresa incorporaba una sección de tejeduría ${ }^{37}$.

Como es fácil suponer, la localización de estas primeras hilaturas mecánicas vino sobre todo condicionada por la energía. Su implantación territorial estuvo en función de la disponibilidad de cursos fluviales y también de la existencia de infraestructuras previas, como molinos laneros, harineros o papeleros, que pudieran ser adaptadas sin excesivo coste. De ahí, que las encontremos en poblaciones atravesadas por ríos, como Manresa, Olot, Ripoll, Vic, Sabadell y Martorell, o que tenían acequias o canales con caudal suficiente para mover algunas máquinas, como era el caso de Barcelona ${ }^{38}$. La geografía, por tanto, de la water fue mucho más restringida que la de jenny por sus requerimientos energéticos y de inversión. De hecho, sólo arraigó de forma destacada en Manresa ${ }^{39}$. La capital del Bages concentraba en 1807 casi la mitad de los husos de water que había en Cataluña y en 1841 esa proporción había subido

35 En algunas ciudades, como Barcelona, donde las posibilidades de utilizar la energía hidráulica eran mínimas, las water-frames también se movieron por caballerías mediante norias, que en Cataluña eran denominadas vogis. Como ha señalado Angels Solà, en Manresa estas máquinas se podían mover a mano mediante unas palancas especiales cuando el caudal del río disminuía hasta el punto de impedir su normal funcionamiento. Solà (2004a), p. 77.

36 García Balañà (2004), pp. 213-214.

37 Una parte significativa de las primeras hilaturas mecánicas movidas por agua tenían también jennies. Entre ellas, podemos citar los casos de Serra, Torruella y Compañía de Barcelona, que en 1799 tenían 23 "máquinas inglesas" y 35 "simples"; de Codina, Dalmau, Martí y Serrano que en una de sus fábricas de Manresa tenían, en 1806, 19 "máquinas inglesas" y 32 "sencillas"; o de Joan Vilaregut, que en 1807 tenía dos fábricas, una en Martorell con 18 "máquinas inglesas" y otra en Barcelona con 46 "simples" y 100 telares.

38 En Barcelona, la única agua que se podía utilizar para mover máquinas era la del Rech Comptal, un canal de escaso caudal que no permitía grandes usos energéticos, sólo algunas ruedas de escasa potencia. Por ello, uno de los principales fabricantes de hilados de la ciudad, Jacinto Ramon, que fue el primero en instalar una máquina de vapor en 1805, la utilizó para bombear agua del Rech con la cuál mover después sus "máquinas inglesas". Agustí i Cullell (1983), pp. 105-133. Estas limitaciones hicieron que la mayoría de las water-frames instaladas en Barcelona se movieran por medio de caballerías (vogis).

39 En el caso de Manresa, también las disponibilidades de capital y las características del mercado desempeñaron un papel importante. Como ha demostrado Llorenç Ferrer, el desarrollo durante el siglo XVIII de una potente industria sedera que exportaba a América una parte considerable de su producción había permitido acumular el capital suficiente como para aportar, sin ayuda exterior significativa, la inversión necesaria para crear hilaturas mecánicas. Unas fábricas que producían justamente el tipo de hilo de urdimbre que necesitaba para sus telares manuales de remo una especialidad textil -la cintería- que se había ido consolidando en las últimas décadas del siglo y en las que participaban también los empresarios del sector de la seda. Esta conjunción no era una casualidad. Los impulsores de las fábricas de Manresa fueron precisamente sederos y cinteros de la ciudad, que no sólo conocían el mercado local sino que eran a su vez clientes de sus propias empresas. La imbricación entre tipo de tecnología y mercado local para explicar la localización de estas primeras hilaturas mecánicas se dio también, aunque con menor intensidad, en otras poblaciones donde existían especialidades propias, como Olot con las medias y gorros de algodón o Vic con las mantas. Sobre la cintería manresana, Ferrer (1994). 
al 78\%. Entre esas mismas fechas el número total de máquinas continuas en Cataluña había aumentado sólo en un tercio (de unas 230 a 289), aunque el número de husos se había más que doblado (de cerca de 11.000 a casi 29.000). La mayor capacidad de las máquinas en 1841 lo explica. Pero estos incrementos muestran claramente el discreto desarrollo que tuvo esta tecnología, en comparación con las otras dos, durante la primera mitad del siglo XIX.

Una de las razones que explican ese escaso desarrollo es que desde 1815 existía un sistema tecnológico que podía ser alternativo, ya que era mucho más productivo a igualdad de $\operatorname{coste}^{40}$. En efecto, la mule, como hemos visto anteriormente, fue la última de las máquinas de hilar que hizo su aparición en Cataluña y su difusión sólo se produjo después de la Guerra de la Independencia. Pero lo hizo con rapidez, aunque limitada básicamente a aquellas zonas donde esta tecnología tenía ventajas comparativas. De hecho, hasta los años treinta su principal, pero no única, localización fue Barcelona, donde, en 1829, el número de mules ya superaba claramente al de jennies y bergadanas juntas — 410 frente a $323-{ }^{41}$.

Las causas por las que esta tecnología tendió a localizarse preferentemente en Barcelona tienen que ver, sobre todo, con las características técnicas de la máquina y sus requerimientos energéticos, el tipo de hilo que producía y el tamaño del mercado ${ }^{42}$. La mule era una máquina de carro que, aunque de mayor tamaño que las anteriores - las habituales eran de 120 husos, pero las había también de 240 husos-, tenía la ventaja de que consumía menos energía que la water-frame y podía ser movida tanto por caballerías como por agua. Era, además, una máquina muy versátil desde un punto de vista productivo, ya que podía producir diversos tipos de hilos, tanto para trama como para urdimbre, y del tamaño requerido, tanto hilos gruesos como finos. Estaba, por tanto, en condiciones de atender una demanda más amplia y exigente, pues permitía fabricar tejidos de mayor calidad que las tradicionales empesas. Y, podía, finalmente, adaptarse sin graves problemas a las formas de organización del trabajo imperantes, ya que tanto podía utilizarse en pequeños talleres como en fábricas de mayor envergadura.

40 Las ventajas de la mule sobre la water-frame en Tunzelman (1978) y Cohen (1985). Según datos de este último autor, en la década de 1830 había en Gran Bretaña 12 husos de mule por cada uno de continua. Una proporción idéntica se daba en Cataluña en 1841.

41 Fuera de Barcelona existieron fábricas de río que utilizaban mule-jennies durante el primer tercio del siglo XIX, pero es muy difícil distinguirlas de las que empleaban water-frames y no es posible, por tanto, establecer su localización específica. En 1833, había en Cataluña, según la estadística de la industria algodonera realizada por Magín Corominas por encargo de la Junta de Comercio, 36 fábricas de hilados movidas por agua, de las cuales una buena parte, sin duda, tenían mule-jennies, pero no sabemos ni cuántas ni cuáles eran. Lo que sí sabemos es que algunas ya estaban equipadas con estas máquinas en 1820, como la que Joan Rull tenía en Sta. Eugenia, un pueblo próximo a Girona, donde tenía una mule de 144 husos, cinco de 216 y dos de 240, además de seis continuas que iban de los 112 a los 168 husos. Cabe pensar que fuera de Barcelona también había fábricas que utilizaban mule-jennies movidas por energía animal, pero la ausencia de datos al respecto permite formular la hipótesis de que en esta primera fase del proceso de industrialización la mule-jenny sólo compitiera favorablemente con las otras máquinas si era movida por agua y estaba instalada en empresas de cierta dimensión (por eso, había ya más husos de mule-jenny que de waterframe en el conjunto de Cataluña desde, al menos, 1829) y que, por ello, fueran escasas las fábricas pequeñas movidas por motores de sangre, porque éstas no representaban ninguna ventaja sustancial sobre los talleres de utilizaban jennies.

42 En Barcelona coexistieron durante los primeros años del siglo XIX los tres sistemas tecnológicos. Pero después de la Guerra de la Independencia la hilatura con máquinas continuas prácticamente desapareció, mientras que la basada en la jenny y en la bergadana fue perdiendo paulatinamente terreno frente a la de la mule-jenny. 
Estas características la hacían idónea para una ciudad como Barcelona, donde se podía utilizar sin restricciones la energía animal, el gran tamaño del mercado permitía desarrollar economías de aglomeración y no faltaban ni capital ni mano de obra cualificada. Por ello, su desarrollo fue tan intenso. Los 1.770 husos de mule que había en la ciudad en 1807, se habían convertido en casi 50.000 en 1829. Sin embargo, hasta la renovación tecnológica de la década de los treinta, la mule no alteró los parámetros organizativos que se habían establecido en la ciudad a principios de siglo. Estos continuaban basándose en empresas de reducidas dimensiones con pocas máquinas y escasos trabajadores. En 1829, de las 50 fábricas que utilizaban mules en Barcelona, sólo 16 tenían más de diez máquinas, 23 oscilaban entre 5 y 9 y las 11 restantes tenían menos de 5, lo que daba una media de 8,2 por empresa.

En definitiva, la tecnología mule había arraigado en la capital gracias sobre todo a las ventajas derivadas del tamaño del mercado y de la abundancia de capital y trabajo. Pero su crecimiento presentaba una importante limitación. El tipo de motor utilizado -las caballeríasimpedía crear fábricas de gran dimensión y hacía imposible con ello desarrollar economías de escala y procesos de integración vertical ${ }^{43}$. Tendía, en consecuencia, a perpetuar la estructura organizativa tradicional heredada de la hilatura manual, al tiempo que era un obstáculo casi insalvable para poder seguir avanzando en el proceso de innovación tecnológica justo cuando empezaban a ser introducidas las nuevas mules y los telares mecánicos. Sustituir la energía animal por la del vapor se convirtió en lo sucesivo en una necesidad vital para los fabricantes de Barcelona.

\section{La apuesta por la energía del vapor y la preponderancia de las ciudades del litoral (1833-1857)}

El mapa de la hilatura del algodón experimentó cambios profundos entre los años 1833 y 1857. Estas modificaciones se inscriben en el marco de una nueva coyuntura económica. A partir de 1826, la unificación fiscal del territorio español y la política aduanera proteccionista de Fernando VII facilitaron la integración de la industria algodonera catalana en un mercado nacional cada vez más protegido de la competencia extranjera ${ }^{44}$. A fin de asegurarse ese mercado, los empresarios buscaron reducir los precios de venta de sus productos modernizando sus equipamientos. Rápidamente, se encontraron enfrentados al problema de la energía disponible para accionar las nuevas máquinas. Este proceso de modernización puso en cuestión la capacidad de los territorios tradicionales de la hilatura para acoger las innovaciones tecnológicas.

\subsection{El tiempo de los grandes cambios tecnológicos}

Tres décadas fueron necesarias para que la mecanización de la hilatura del algodón se completara en Cataluña ${ }^{45}$. Las bergadanas y las mule-jennies de madera cedieron su lugar a máquinas más perfeccionadas: la versión mejorada de la water-frame adoptada en 1832-1833, los nuevos modelos de la mule-jenny que se difundieron en el curso de los años treinta y la selfac-

\footnotetext{
43 Sánchez (1996).

44 Sánchez (2000a).

45 Para una visión general del proceso. Maluquer de Motes (1976) y Nadal (1991).
} 
tina introducida en $1844^{46}$. En 1850, como se puede apreciar en el cuadro 3, la hilatura estaba ya totalmente mecanizada en Barcelona. Para el conjunto de Cataluña, el proceso fue algo más lento, pero se había completado ya a finales de esa misma década.

CUADRO 3

NÚMERO DE HUSOS DE HILAR EN BARCELONA Y CATALUÑA ENTRE 1807 Y 1885

\begin{tabular}{cccccc}
\hline Años & Barcelona & $\begin{array}{c}\% \text { de } \\
\text { mecanización } \\
\text { Barcelona }\end{array}$ & Cataluña & $\begin{array}{c}\text { \% de } \\
\text { mecanización } \\
\text { Cataluña }\end{array}$ & $\begin{array}{c}\text { Barcelona/ } \\
\text { Cataluña } \\
\%\end{array}$ \\
\hline $\mathbf{1 8 0 7}$ & 24.774 & 15,4 & 95.890 & 13,6 & 25,8 \\
$\mathbf{1 8 2 9}$ & 78.270 & 63 & 386.013 & 21 & 20,3 \\
$\mathbf{1 8 3 6}$ & 160.017 & 80 & 651.047 & 29,7 & 24,6 \\
$\mathbf{1 8 4 1}$ & 226.849 & 90 & 792.723 & 43,6 & 28,6 \\
$\mathbf{1 8 5 0}$ & 242.876 & 100 & 806.636 & 77,2 & 30,1 \\
$\mathbf{1 8 5 7}$ & 174.216 & 100 & 896.064 & 99,5 & 19,4 \\
$\mathbf{1 8 6 1}$ & 166.801 & 100 & 770.417 & 99,9 & 21,7 \\
$\mathbf{1 8 6 5}$ & 135.451 & 100 & $?$ & 100 & $?$ \\
$\mathbf{1 8 7 1}$ & $?$ & 100 & 1.200 .000 & 100 & $?$ \\
$\mathbf{1 8 7 5}$ & 113.676 & 100 & $?$ & 100 & $?$ \\
$\mathbf{1 8 8 5}$ & 122.130 & 100 & $1.900 .000^{*}$ & 100 & 6,4 \\
\hline
\end{tabular}

Nota: * Cifra de 1884.

Fuentes: Arxiu Administratiu de Barcelona, A 1.232; Amorós (1857); Graell (1911); Maluquer de Motes (1976), p. 136; Nadal et Tafunell (1992); Sánchez (2000a), pp. 506-508; de Sard (1884), pp. 26-27.

Estas innovaciones tuvieron importantes repercusiones en el terreno energético. Hechas de hierro y dotadas de un número de husos en constante aumento, estas nuevas máquinas eran más pesadas y requerían mucha más energía para funcionar. Para reemplazar a la fuerza humana o animal, los empresarios de la hilatura recurrieron tanto a la energía hidráulica como a la del vapor. El desarrollo del uso de la rueda hidraúlica se inscribe, como hemos visto, en la dinámica del periodo anterior. No obstante, entre 1829 y 1856, como muestra el cuadro 4, la potencia de la energía hidráulica puesta al servicio de la hilatura del algodón se multiplicó por diez. En 1833, la empresa barcelonesa Bonaplata,Vilaregut, Rull y Cía. instaló la primera máquina de vapor. Veintitres años después, en 1856, la fuerza motriz de los aparatos a vapor que funcionaban en las fábricas de hilados catalanas doblaba a la generada por las ruedas hidráulicas. Indudablemente, los años 1833-1857 estuvieron caracterizados por el triunfo del vapor.

Los condicionantes energéticos de esta primera industrialización provocaron una profunda reorganización de la geografía de la producción. Este proceso se articuló en torno a un triple movimiento. Por una parte, los territorios de la bergadana y del trabajo a domicilio, como las comarcas de l'Anoia y del Berguedà, desaparecen rápidamente de los primeros lugares de las estadísticas (Cuadro 5). ¿Significa eso que el interior de Cataluña se vio afectado globalmente por el retroceso de la hilatura del algodón? Sólo en parte, ya que la segunda característica de este proceso de reorganización fue la importancia que adquirieron ciertas zonas alejadas del litoral. Aquellas, en especial, que utilizaban tradicionalmente la energía hidráulica —sobre

46 Sobre estas innovaciones. Ferrer (2004); Solà (2004a, 2004b) y Thomson (2003a). 
CUADRO 4

FUERZA MOTRIZ HIDRÁULICA Y DE VAPOR EN LA HILATURA DE ALGODÓN EN CATALUÑA ENTRE 1807 Y 1911 (POTENCIA EN CABALLOS DE VAPOR)

\begin{tabular}{ccccc}
\hline Años & $\begin{array}{c}\text { Motores } \\
\text { hidráulicos }\end{array}$ & $\begin{array}{c}\text { Máquinas } \\
\text { de vapor }\end{array}$ & Total & \% Vapor \\
\hline $\mathbf{1 8 0 7}$ & 39 & - & 39 & - \\
$\mathbf{1 8 2 9}$ & 186 & - & 186 & - \\
$\mathbf{1 8 3 2}$ & $?$ & 30 & $?$ & $?$ \\
$\mathbf{1 8 4 1}$ & 565 & 300 & 865 & 52,69 \\
$\mathbf{1 8 5 0}$ & 1.660 & 1.852 & 3.512 & 64,54 \\
$\mathbf{1 8 5 6}$ & 1.716 & 3.123 & 4.839 & 64,36 \\
$\mathbf{1 8 6 0}$ & 2.067 & 3.733 & 5.800 & 25 \\
$\mathbf{1 9 1 1}$ & 26.727 & 8.909 & 35.636 & \\
\hline
\end{tabular}

Fuentes: Carreras (1983), p. 42; Nadal (1991), pp. 28-51; Rosés (2001b), p. 10; Sánchez (2000a), p. 508.

todo el curso medio del río Llobregat- y que vieron ahora reforzado su papel "motor" gracias al gran impulso del proceso de mecanización. El tercer componente de esta reorganización territorial fue la confirmación de Barcelona y su "interland" como la principal área productiva de hilados de algodón de Cataluña. El liderazgo de la capital se fundamentó sobre todo en la rápida e intensa adopción de la energía del vapor y sirvió, además, como modelo para otras ciudades del litoral, que se engancharon en estos años a la vía de industrialización "a la inglesa". En definitiva, dos ámbitos territoriales y energéticos bien distintos acabaron constituyendo la nueva geografía de la hilatura del algodón en los años 1833-1857.

\subsection{La fábrica de río: ¿un modelo al borde de la saturación?}

Aunque la energía hidráulica estaba sometida al régimen caprichoso de los cursos de agua catalanes, fue una fuerza motriz rentable en términos de costes de funcionamiento. Por ello, los fabricantes de hilados no dejaron de utilizarla ampliamente durante la primera mitad del siglo XIX. Gracias a las leyes y decretos que pusieron fin definitivamente al régimen señorial de las aguas y atribuyeron ciertos privilegios fiscales a los empresarios, los años 1830-1840 fueron propicios a la instalación de hilaturas hidráulicas ${ }^{47}$. En 1833 ya había $36^{48}$. En 1856, como puede verse en el mapa 1, el número se había más que doblado hasta llegar a 79. Este desarrollo se produjo sobre todo en los cursos medios del Ter y del Llobregat y confirmó la zona de Manresa como una de las más importantes de Cataluña en el uso de la energía hidráulica ${ }^{49}$. Por tanto, estas fábricas "sobre el agua" se instalaron a una distancia de Barcelona que, en términos de coste del transporte, puede considerarse razonable.

47 Dorel-Ferré (1999); Maluquer de Motes (1990) y Clua (2001). La Real Orden de 14 de marzo de 1846 declaraba a las fábricas hidráulicas exentas del pago de la mitad de la contribución fiscal por un plazo de 10 años.

48 Gutiérrez (1837), pp. 174-175.

49 En 1841, concentraba ella sola casi la mitad de la potencia hidráulica total de las hilaturas catalanas. Ferrer (2004), p. 353. 


\section{MAPA 1}

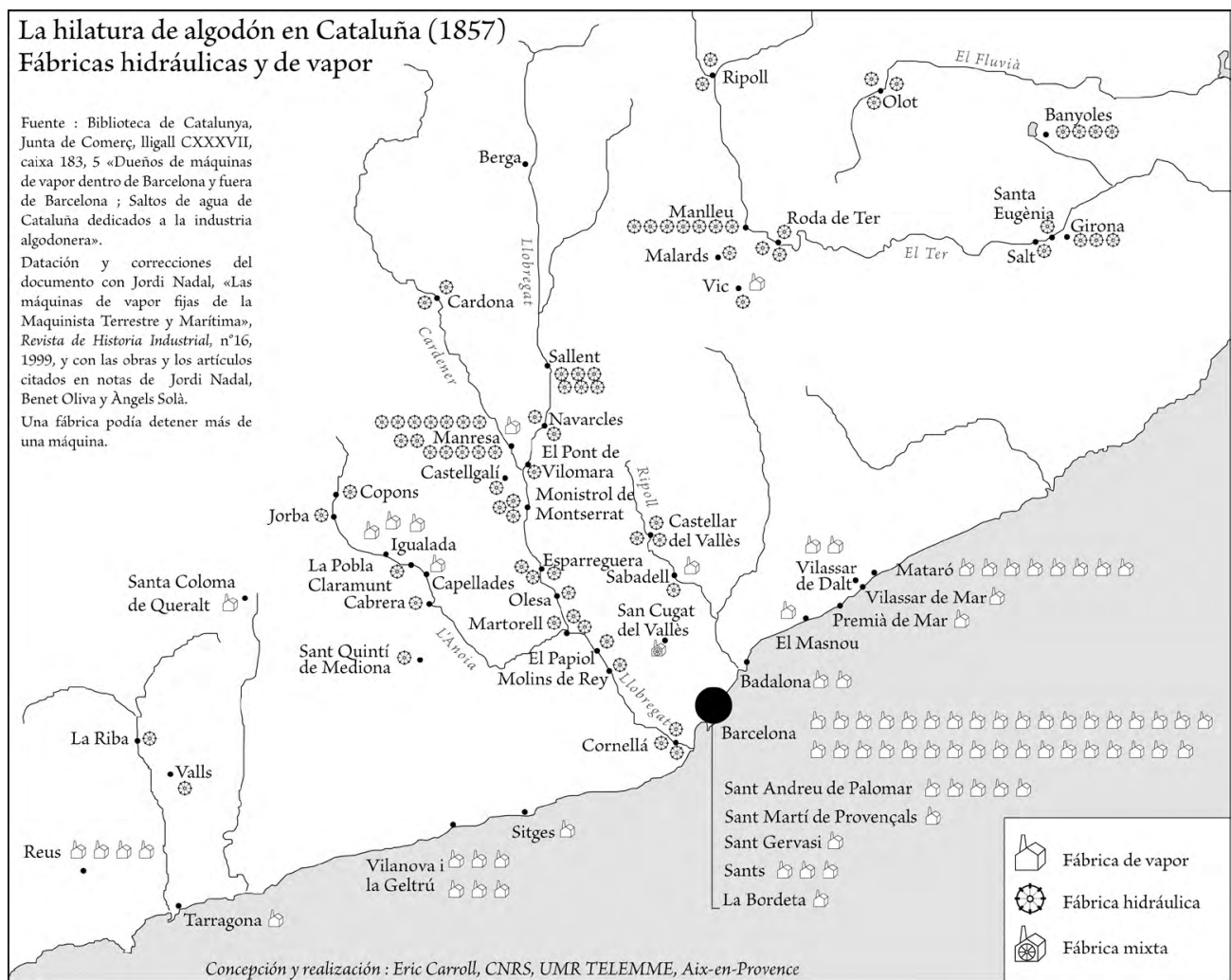

Las razones de esta localización son diversas. En los años 1830 fueron principalmente políticas y militares, debido a que la primera guerra carlista paralizó buena parte de las actividades industriales en las zonas más interiores de Cataluña. También hay que considerar el tema de los costes del transporte. En ausencia del ferrocarril y con una red mediocre de carreteras, un alejamiento excesivo de Barcelona penalizaba las empresas y anulaba los beneficios derivados del uso de la energía hidráulica ${ }^{50}$. La última razón reside, como ya hemos visto, en las ventajas heredadas de la geografía industrial tradicional, con la presencia antigua, a lo largo de los cursos de agua y de los canales, de molinos harineros, fábricas de papel y establecimientos ligados al trabajo de la lana y de la seda. Esta herencia fue determinante ya que si la energía hidráulica es poco costosa en su funcionamiento es cara en lo que se refiere a su instalación. Para evitar los gastos ligados a la construcción de edificios y a la instalación de los saltos de agua, los empresarios se beneficiaron, hasta finales de los años 1840, de la existencia de antiguos molinos, que fueron comprados o alquilados en parte o en su totalidad ${ }^{51}$.

$50 \quad$ Maluquer de Motes (1990), p. 335 y Cuadro 4.

51 Un buen estudio sobre el valle del río Ripoll: Benaul y Deu (2005), pp. 176-177. 
La energía hidráulica tuvo en estos años un gran éxito, pero éste, sin embargo, no fue duradero. La década de 1850 se caracteriza por un estancamiento en su uso. La rápida ocupación de los mejores emplazamientos supuso la saturación de estos espacios productivos ${ }^{52}$. El estancamiento de la oferta de saltos de agua se convirtió, así, en un problema, que sólo en muy pequeña medida pudo ser paliado por la construcción de canales colectivos ${ }^{53}$. Desde mediados de los años 1840, la competencia entre empresarios por la utilización de las aguas de los ríos se hizo feroz ${ }^{54}$. Para los fabricantes de hilados la situación se vio agravada por el volumen de la inversión necesaria para lanzarse a la aventura industrial. Los molinos disponibles eran muy escasos y era necesario construir establecimientos nuevos, adecuar los emplazamientos, e incluso comprar una máquina de vapor para asegurar la continuidad de la producción cuando el caudal de los ríos era bajo. En términos de costes y de posibilidades de instalación la situación había cambiado. El crecimiento se vio bloqueado por la sobreexplotación de los territorios tradicionalmente detentadores de recursos hidráulicos.

\subsection{El papel motor del distrito industrial de Barcelona y de las ciudades del litoral}

Si la energía hidráulica fue preponderante en los inicios de la modernización de la hilatura, la difusión de la energía del vapor en los años cuarenta situó a la industria algodonera catalana en un modelo energético centrado en el carbón. Aparato más potente y regular que la rueda hidráulica, la máquina de vapor permitía incrementar la productividad del trabajo y el rendimiento de la maquinaria. Ella fue entonces la solución a los imperativos técnicos y energéticos del momento ${ }^{55}$. Las cifras muestran la preponderancia del nuevo aparato. Si la primera máquina de vapor entró en funcionamiento en 1833, nueve años después eran 24 y en 1857 ya ascendían a 83 (Mapa 1$)^{56}$. A finales de los años cincuenta, al menos las tres cuartas partes de los husos de la industria algodonera catalana eran accionadas por el vapor $^{57}$.

Esta situación puede parecer sorprendente a primera vista. Desde el decenio de 1830, la industria catalana tuvo que enfrentarse al problema del aprovisionamiento de combustibles minerales porque la ausencia de producción local la obligó a pagar el carbón muy caro, debido tanto a la lejanía de los principales centros de producción españoles como a los elevados derechos de aduanas que gravaban la hulla importada de Gran Bretaña. Las razones del éxito de la energía del vapor tienen que ver a la vez con ventajas inmediatas y retos de futuro. Evidentemente, la saturación de los espacios que permitían el uso a bajo coste de la energía hidráulica, como acabamos de ver, favorecieron la apuesta por el modelo de desarrollo "a la inglesa". Pero, al mismo tiempo, las ventajas de la adopción del vapor eran considerables. La instalación de estos motores permitía la concentración de empresas en Barcelona y en el lito-

\footnotetext{
52 Solà (2004a), p. 246.

53 El único que funcionó fue el canal de Manlleu, construido entre 1841 y 1848.

54 Así, en 1846, Miquel Puig tuvo que enviar a su hermano Cristóbal a Madrid para defender su proyecto de fábrica en Esparraguera frente a otros competidores: Dorel-Ferré (1999), p. 1058.

Sánchez (2000a).

Ferrer (1846), p. 225 y Mapa 1.

Rosés (2001a), p. 96.
} 
ral y ofrecía la posibilidad de disfrutar de las ventajas de la proximidad y la complementariedad. La formación de un distrito industrial en Barcelona en los años 1830-1840 es la mejor prueba de esta situación ${ }^{58}$. También es cierto que esta opción energética se basó en la esperanza de que Cataluña pudiera asegurar, en un futuro próximo, una producción local capaz de sustituir las importaciones de carbón británico. Desde los años 1830, el subsuelo catalán se consideró que era susceptible de albergar carbones de calidad. Por eso, en las dos décadas siguientes se dio una verdadera fiebre minera y los primeros resultados de la explotación de los yacimientos, aunque decepcionantes, no redujeron el optimismo de los empresarios ${ }^{59}$. Entre 1849 y 1861, se presentaron desde Barcelona 117 solicitudes de concesiones a la Junta Facultativa de Minas ${ }^{60}$.

La opción del vapor dictó la localización de una buena parte de las fábricas de hilados catalanas. El litoral se impuso porque era el punto de llegada de los carbones británicos. En esta zona costera, Barcelona fue clave, hasta el punto de que, en los años treinta y cuarenta, el modelo barcelonés fue emulado por poblaciones como Mataró, Vilassar de Mar, Badalona, Sitges, Vilanova i la Geltrú y Reus, que se consolidaron como importantes centros de producción (Mapa 1). Justo a principios de la década de 1860, como muestra el Cuadro 5, tres de las cuatro comarcas litorales de la provincia de Barcelona reunían ellas solas la mitad de los husos catalanes. Este sistema territorial y tecnológico asumió un papel motor en la modernización de la hilatura. Sin embargo, pronto conoció sus limitaciones. La principal vino dada por la factura energética. Los yacimientos locales sólo proporcionaron carbones de mediocre calidad y la precariedad de la red ferroviaria no permitía asegurar todavía una fluida relación entre las minas y los centros fabriles de consumo ${ }^{61}$. La industria algodonera continuó, así, pagando caro su carbón. Pero también las limitaciones vinieron desde el ámbito social y tecnológico. Fue en las grandes ciudades, especialmente en las del litoral, donde surgió el movimiento obrero. Las organizaciones de trabajadores lucharon a menudo contra la introducción de innovaciones técnicas y a favor de la organización tradicional del trabajo. En Barcelona, por ejemplo, la introducción de la selfactina se retrasó por la oposición de las asociaciones obreras, que preferían las mule-jennies porque permitían salvaguardar el empleo masculino, mantener salarios más elevados y asegurar el control de los obreros masculinos sobre los sistemas de trabajo y formación que imperaban en las fábricas ${ }^{62}$. La "cuestión social" se convirtió, así, en un aspecto clave de la dinámica industrial, ya que una parte importante de sus primeras manifestaciones conflictivas estuvieron directamente relacionadas con la innovación tecnológica. El "ludismo" también estuvo presente en los inicios de la industrialización catalana y se manifestó en su doble vertiente destructora (de máquinas) y coactiva (de empresarios) ${ }^{63}$.

58 Raveux (2005).

59 Por ejemplo, las esperanzas de Gerónimo Ferrer y los directivos de La España Industrial en la segunda mitad de los años 1840. Ferrer (1846), p. 235 y Gutiérrez (1997), p. 50.

$60 \quad$ Nadal (1975), p. 153.

61 Los yacimientos de lignitos estaban bastante lejos del litoral (en las comarcas de l'Anoia y la Segarra), al igual que los de hulla, más importantes, que se localizaban en el pre-Pirineo (Sant Joan de les Abadesses).

62 García Balañà (2002), p. 62 y ss.

63 El incendio de la fábrica Bonaplata en 1835, las huelgas durante la regencia de Espartero y el conflicto de las selfactinas en el verano de 1854, son ejemplos de ello. Benet y Martí (1976) y Maluquer de Motes (1976). 


\section{CUADRO 5}

PRINCIPALES COMARCAS POR EL NÚMERO DE HUSOS DE HILAR EN CATALUÑA ENTRE 1807 Y 1900

\begin{tabular}{lllrlrllc}
\hline \multicolumn{1}{c}{1807} & \multicolumn{3}{c}{$1841^{*}$} & \multicolumn{2}{c}{1861} & \multicolumn{2}{c}{1900} \\
\hline Barcelonès & 26,4 & Anoia & 26,6 & Barcelonès & 34,9 & Barcelonès & 23,2 \\
Baix Camp & 19,2 & Barcelonès & 21,4 & Bages & 15,3 & Bages & 22,6 \\
Bages & 13,6 & Berguedà & 15,7 & Osona & 8,1 & Osona & 15 \\
Garrotas & 11,8 & Bages & 11,9 & Garraf & 7,5 & Garraf & 6,6 \\
Osona & 7,5 & Osona & 5,6 & Maresme & 7,4 & Berguedà & 6 \\
\hline
\end{tabular}

Nota: * Para 1841, las cifras se refieren a los partidos judiciales de Igualada, Barcelona, Berga, Manresa y Vich.

Fuentes: Calvo (2002), p. 98; Madoz (1846), p. 462-465; Nadal (1991), p. 54.

\section{La revalorización de la energía hidráulica y de las comarcas del interior (1858-1885)}

El final del decenio de 1850 significó un verdadero cruce de caminos para la hilatura del algodón en Cataluña. La implantación litoral sobre la base de la opción por la energía del vapor no acabó de imponerse del todo. Condicionada por el precio elevado del carbón y las difíciles relaciones entre patrones y obreros, el sistema territorial y técnico de los años 18331857 no pudo mantener el ritmo de crecimiento. Por el contrario, la energía hidráulica, renovada técnicamente, apareció de nuevo como una opción seductora.

\subsection{Nuevas oportunidades y viejos problemas}

Nuevos cambios tecnológicos tuvieron lugar durante la década de 1850, pero, contrariamente al periodo precedente, afectaron poco a las máquinas de hilar. Después de 1856, la selfactina se impuso y su reinado se alargó hasta los años $1880^{64}$. Las principales innovaciones se dieron, sobre todo, en los dominios del transporte y de la energía. El primer impulso de los ferrocarriles catalanes tuvo lugar entre 1854 y 1859. Las poblaciones de Granollers y Manresa fueron entonces unidas por vía férrea a Barcelona y los costes del transporte hacia las cuencas de los ríos Llobregat y Ter disminuyeron sustancialmente ${ }^{65}$. En las tres décadas siguientes, la construcción de nuevas líneas hacia el interior del Principado se hizo realidad. En 1876, el tren llegó a Vic, en 1879 a Manlleu, en 1880 a Ripoll, en 1884 a Sallent y en 1885 a Puig-reig. Con ello, los industriales del algodón tuvieron menos dificultades para remontar los cursos del Llobregat y el Ter y encontrar nuevos emplazamientos para sus fábricas.

Paralelamente, importantes innovaciones se dieron también en el terreno de la energía. En 1847, la fábrica Quer de Sallent instalaba la primera turbina Fontaine y este nuevo motor

64 Es entonces cuando la máquina de hilar contínua de anillas (ring-throstle) inicia su desarrollo. Los primeros aparatos se instalaron en la fábrica Batlló de Les Corts de Sarrià en 1878. Calvo (1999), p. 1168.

65 Paralelamente, durante los años 1850-1860, la red viaria mejoró gracias a los trabajos llevados a cabo por la Junta de Carreteras de Cataluña. Sobre este tema: Pascual (1991, 1999). 
hidráulico se difundió en la segunda mitad de los años $1850^{66}$. El acontecimiento es importante. La turbina Fontaine se adaptaba bien al régimen irregular de los cursos de agua catalanes y permitía mejorar tanto la potencia como la fiabilidad de los motores hidráulicos ${ }^{67}$. Su éxito fue rápido y se vio favorecido por la creación de la empresa Planas, Barné, Junoy y Cía. en Gerona, titular desde 1858 de la patente de su construcción en España ${ }^{68}$.

Estas dos innovaciones ofrecieron nuevas oportunidades a la hilatura y permitieron -o al menos es lo que pensaban muchos empresarios- aportar soluciones a los problemas que pesaban sobre su actividad. En primer lugar, el de la factura energética de las fábricas que funcionaban con carbón. A partir de 1860, el modelo de desarrollo "a la inglesa" parecía abocado a un declive indudable, sobre todo cuando se hizo evidente que no existía en Cataluña ningún yacimiento carbonífero susceptible de proporcionar productos de calidad a precios razonables ${ }^{69}$. El carbón se había convertido, según la conocida expresión de Jordi Nadal, en el verdadero "talón de Aquiles de la industrialización catalana" y la turbina fue entonces un balón de oxígeno ya que permitió volver a la energía hidráulica en condiciones competitivas ${ }^{70}$.

Pero estas innovaciones permitieron también a los empresarios enfrentar el problema cada vez más grave de las relaciones con sus obreros. Desde que la situación política lo permitió, las organizaciones de trabajadores se mostraron cada vez más activas en su enfrentamiento con los empresarios. Durante el Bienio Progresista (1854-1856) y también en el Sexenio Revolucionario (1868-1873), las huelgas fueron muy numerosas tanto en Barcelona y su área de influencia como en las poblaciones situadas en las riberas del Llobregat y del Ter. Los obreros del algodón no cesaron de luchar contra la feminización del trabajo del hilado, que era vista como un medio de reducir los costes de fabricación por parte de los empresarios ${ }^{71}$. Para acabar con estos conflictos en el medio urbano y poder reclutar una mano de obra más barata y, sobre todo, más dócil, los fabricantes de hilados consideraron también la opción, abierta ahora por las nuevas posibilidades que ofrecía la energía hidráulica, de buscar nuevos emplazamientos para sus industrias en las cuencas fluviales del interior del Principado, alejadas de los centros urbanos.

\subsection{El lento declive de los espacios litorales}

La renovada atracción por las zonas del interior y la energía hidráulica puso de relieve el tema del declive del litoral. En Barcelona el fenómeno es bien visible. La capital tenía en funcionamiento más de 225.000 husos en $1855^{72}$. A finales de los años 1880 se habían reducido a poco más de 60.000. Desde este punto de vista, Barcelona ocupaba ahora una posición marginal en el sector de la hilatura en Cataluña. ¿Era esta una situación específica de la capital o un fenómeno más general que afectaba al conjunto de las localidades costeras? Si juzgamos por el nivel de actividad de poblaciones importantes, como Mataró o Vilanova i la Geltrú, así

\footnotetext{
Concretamente en Manresa. Solà (2004a), pp. 165-166.

Nadal (1985), p. 53 y (1992), p. 63.

Nadal (1992), p. 63. En 1880, la firma de Girona ya había instalado 300 turbinas. Carreras, (1983), p. 46.

Carreras (1983), p. 43.

Nadal (2000), p. 534.

Garcia Balañà (2002), p. 67 y ss.

Nadal y Tafunell (1992), p. 75.
} 
como por la creación de importantes fábricas en los municipios del Llano de Barcelona, es la primera explicación la que se impone, y viene a confirmar la resistencia de la localización litoral y de la utilización del vapor. En la década de 1870, las dos mayores hilaturas de algodón de Cataluña -la fábrica Batlló de Les Corts de Sarrià y La España Industrial de Santsestaban situadas en la periferia de Barcelona ${ }^{73}$.

La persistencia de la localización en el litoral de las fábricas de vapor tiene numerosas explicaciones. Evidentemente, la proximidad de las instalaciones portuarias, el desarrollo, aunque lento, de la red ferroviaria y las ventajas derivadas de la inmersión en un territorio productivo muy denso y próximo al principal mercado de consumo desempeñan un papel muy importante. Hay también otras dos razones y ambas tienen que ver con el carbón. La primera está relacionada con mejoras tecnológicas — como la difusión del sistema de distribución Corliss - que permitió reducir el consumo de combustible de las máquinas de vapor. En 1833, el consumo anual de carbón era de 15 toneladas por caballo de vapor ${ }^{74}$. Esta cifra se redujo a 8,76 toneladas en 1848 y a menos de 6 en el periodo 1882-1885. Las ganancias derivadas de esta reducción se añadieron a las que proporcionaba la disminución del precio del carbón. Los trabajos de Raimon Soler sobre la fábrica de la Rambla de Vilanova y sobre La España Industrial lo demuestran claramente. Entre 1856-1860 y 18811885, el precio del carbón se redujo en más de un tercio, pasando de 55,8 pesetas por tonelada a 34,9, en el caso de la primera, y de 52,6 a 33,8, en la segunda ${ }^{75}$. Este fenómeno fue particularmente significativo a partir de 1863, gracias a la reducción del precio del transporte marítimo y de los derechos de aduanas para los combustibles. Estos dos factores acumularon sus efectos para permitir la disminución sustancial de la parte del combustible en el conjunto de los costes de producción de las empresas. En 1842, el carbón representaba el 6,9\% de los gastos de fabricación en la empresa de la Rambla. La cifra cayó al 3,7\% en 1875 .

¿Pero, incluso en estas condiciones, podía el vapor resistir aún mucho tiempo? El periodo de recesión económica que se inició en 1883 parece señalar el fin de su reinado. La crisis de la Bolsa, con su secuela de quiebras empresariales, la firma de los tratados comerciales con Francia, Alemania e Inglaterra que colocaron a España en la senda del libre-cambio y la contracción del mercado nacional, ya de por sí poco elástico, como consecuencia de las malas cosechas agrícolas, repercutieron sobre la industria algodonera catalana provocando el cierre de numerosas fábricas ${ }^{76}$. Los establecimientos a vapor del litoral fueron los primeros en sufrir las consecuencias. Para los empresarios, la reducción de costes de producción se convirtió entonces en una cuestión de supervivencia. Hacia 1890, el 53\% de los obreros del algodón trabajaban ya en fábricas de río del interior del país ${ }^{77}$. La energía hidráulica parecía en aquellos momentos haber ganado la partida y vuelto a redefinir el mapa de la hilatura algodonera catalana.

73 Estas dos fábricas contaban respectivamente con 53.000 y 42.000 husos en 1888. Enrech (2003), p. 19.

74 Los cálculos han sido efectuados sobre la base de 300 días de trabajo por año y 12 horas de funcionamiento al día de las máquinas (de 15 caballos). Para 1833 ver Gutiérrez (1837), p. 176; para 1848 Figuerola, (1849), p. 291; para la primera mitad de los años 1860, Información, IV, p. 89; y para 1882-1885, Soler (2001a), pp. 97-98. Esta última cifra es una aproximación a partir de los datos concernientes a dos máquinas Corliss de la fábrica de la Rambla de Vilanova i la Geltrú.

75 Los datos de estas dos empresas en Soler (2001b), pp. 3-4.

76 Enrech (2003), p. 51.

77 Enrech (2001), p. 573, y (2005) para una visión más general. 


\subsection{De la fábrica de río a las colonias industriales}

Durante el periodo 1858-1885, la renovación de la energía hidráulica, al provocar un aumento sustancial de la fuerza motriz empleada en los establecimientos situados sobre los cursos de agua, favoreció un descenso significativo de los costes de producción ${ }^{78}$. Éste éxito fue el resultado de la voluntad de los empresarios de aplicar las innovaciones tecnológicas. Pero también el fruto de su apropiada gestión del problema de la localización fabril. Desde mediados de los años cuarenta, el reducido número de saltos de agua disponibles y la competencia para alquilar o comprar edificios industriales dificultaron que las áreas geográficas tradicionales que utilizaban la energía hidráulica pudieran continuar desempeñando un papel determinante en la instalación de las nuevas fábricas de hilados. Los empresarios tuvieron entonces que buscar nuevos espacios, que sólo podían encontrar en el medio rural ${ }^{79}$. Fue a partir de ese momento cuando tuvo lugar una reorganización productiva y territorial de la hilatura, que dio lugar a la época de esplendor de las colonias industriales. Éstos verdaderos pueblos de producción, "aglomeraciones autónomas" del municipio al que se adhieren y que "dependen del salto de agua que les provee de energía" ${ }^{80}$, aparecieron tímidamente a finales de los años 1840, aunque se desarrollaron principalmente tras la creación de la colonia Rosal en 1858, acontecimiento emblemático en la génesis del movimiento. A partir de los años 1870, la localización de estos nuevos sistemas territoriales de producción se densificó hacia el alto Llobregat y las fuentes del Ter y el Freser. Hacia 1885, ya eran una treintena las colonias existentes, la mayoría sobre el Llobregat, y la comarca del Berguedà se había convertido en su principal centro de difusión ${ }^{81}$.

¿Cómo explicar el éxito de las colonias industriales? Sin duda, los imperativos de un descenso en los costes de producción están en la base de su desarrollo, aunque semejante elemento no parece suficiente para ofrecer una comprensión completa del fenómeno. Hace falta, para ello, abordar diversos problemas económicos y sociales, algunos de los cuales pueden ser objeto de un análisis bastante preciso. Para empezar, la búsqueda, por parte de los empresarios, de una reducción en los costes del trabajo y de una mayor flexibilidad de la mano de obra era posible con el reclutamiento de los obreros en el medio rural ${ }^{82}$. La creación de las colonias permitió, por otro lado, conseguir otras economías gracias a la concentración de las actividades que facilitó la integración vertical de las empresas ${ }^{83}$. El tema fiscal también desempeñó un papel apreciable. Las importantes exenciones a la contribución fiscal que tuvieron lugar durante el periodo comprendido entre la Real Orden del 14 de marzo de 1846 y la ley del 3 de junio de 1868, fueron un incentivo financiero no despreciable ${ }^{84}$. Éstas, combinadas con la reducción de la factura energética, permitieron compensar los sobrecostes del

78 Según Maluquer de Motes (1990), p. 340, el coste de un caballo hidráulico era la mitad de un caballo de vapor en el último cuarto del siglo XIX.

79 Se pueden ver numerosos ejemplos en Oliva (2000), pp. 415-416 y Solà (2004a), p. 246.

80 Dorel-Ferré (2004), p. 266.

81 El cuadro completo que recoge la cronología de la instalación de las colonias industriales en Cataluña entre 1858 y 1902, en Dorel-Ferré (1992), p. 53.

82 Dorel-Ferré (1999), p. 1063 y Smith (2003), p. 84. La mano de obra era entre un $20 \%$ y un 30\% más barata que en Barcelona y trabajaba más horas por día.

83 Era el caso, por ejemplo, de l'Ametlla de Merola. Esta colonia nació de la fusión de tres establecimientos (los de Balsareny, Vilanova i la Geltrú y Vilassar de Dalt) y permitió reunir en el mismo espacio hilatura y tisaje. La historia de esta empresa en: Oliva (1999), p. 508.

84 Sobre este tema véase: Carreras (1983), p. 55 y Clua (2001). 
transporte que suponía la mayor distancia hasta el principal centro de aprovisionamiento y consumo que era Barcelona.

Quedan, finalmente, dos cuestiones cuyo impacto real es difícil de evaluar: la cuestión obrera y el papel del ferrocarril. Son numerosos los autores que han señalado la importancia que, en la creación de las colonias, ha tenido la voluntad de los empresarios de acabar con los problemas generados por las organizaciones obreras en los centros de trabajo urbanos ${ }^{85}$. Algunos, por el contrario, rechazan esta explicación, aunque reconocen que si bien no existía esta decidida voluntad empresarial de "solucionar" la cuestión obrera mediante el modelo de la colonia, ello "no era incompatible con el ejercicio del paternalismo una vez creada" ésta ${ }^{86}$. Hay, no obstante, elementos que permiten pensar que no eran pocos los empresarios para los que sí había una relación directa entre estos dos temas. Por ejemplo, el hecho de que el primer desarrollo de las colonias se produjo pocos años después de la crisis de las selfactinas del verano de 1854, que puso de manifiesto el tema de la conflictiva sustitución de mano de obra masculina por femenina. Un tema que, desde la óptica empresarial, probablemente podría enfrentarse mejor en el marco de una nueva organización productiva y territorial. Con todo, esta es una cuestión que requiere aún mayor investigación.

Tampoco es fácil explicar el papel desempeñado por el ferrocarril. Desde finales de los años 1870, la industrialización del pre-pirineo se apoyó en el desarrollo de la red ferroviaria. Pero, ¿realmente los caminos de hierro fueron un impulso decisivo en la creación de las colonias industriales? No está claro. Cuando se fundaron las primeras colonias, muchas de ellas en el curso alto del Llobregat, no había ninguna línea férrea que pasara de Manresa. Por ello, puede formularse la hipótesis de si el ferrocarril no fue más una consecuencia de la creación de las colonias industriales que una causa.

\section{Conclusión}

En Cataluña, la diversidad de los territorios industriales y de las organizaciones productivas permitió a la hilatura del algodón desarrollarse mediante una constante adaptación a las cambiantes condiciones económicas, sociales y tecnológicas que se fueron dando durante la primera revolución industrial.

Su primera implantación territorial se produjo a finales del siglo XVIII y estuvo condicionada por el cambio tecnológico que estaba entonces transformando la industria algodonera europea. La localización de la producción no estuvo determinada en aquellos momentos sólo por la existencia de un tejido industrial previamente establecido, sino también por otros factores como la energía y el tamaño del mercado. Durante la primera fase de la industrialización, la localización de la hilatura estuvo estrechamente relacionada con los sistemas tecnológicos surgidos de la utilización de las nuevas máquinas de hilar. De hecho, en cada territorio los empresarios tendieron a adoptar la tecnología que, por sus características específicas, se adaptaba mejor a las estructuras productivas previamente existentes. Así, mientras la jenny, una tecnología intensiva en factor trabajo, se difundía extensamente por el territorio donde la previa implantación de la hilatura manual había ya formado y organizado una mano de obra especializada, la water-frame encontró su territorio junto a los cursos de agua capaces de mover máquinas que requerían mucha energía. Por su parte, la mule-jenny se implantó allí

85 Terrades (1994); Oliva (1999) y Smith (2003).

86 Dorel-Ferré (2005), p. 74. 
donde el tamaño del mercado y las características específicas de la demanda permitían aprovechar mejor su capacidad de producción. Frente a la amplia difusión de la jenny, la utilización de la water-frame se limitó a las zonas fluviales, mientras la mule-jenny se localizaba preferentemente en Barcelona, la única ciudad capaz de generar economías de aglomeración en el primer tercio del siglo XIX.

Los años 1835-1885 se caracterizan por la importancia de las nuevas localizaciones territoriales que aparecen para hacer frente a las necesidades económicas, energéticas y sociales surgidas con la industrialización. Un sistema dual, definido a la vez en función de la implantación territorial (cuencas de los ríos del interior y ciudades portuarias) y de la fuerza motriz utilizada (hidráulica y de vapor), rediseña en estas décadas el mapa de la hilatura en Cataluña. La opción inicial por el vapor en las comarcas del litoral que se lo pueden permitir, parece dejar en un segundo plano a las tradicionales fábricas de río del interior, que, sin embargo, se reivindicarán a partir de la década de 1850. ¿Por qué se produjo ese movimiento pendular? ¿Fue el triunfo de la racionalidad económica para producir a menor coste? Sin duda, pero no sólo. El declive de la hilatura en Barcelona y en las comarcas litorales y el éxito de las colonias industriales no se explican únicamente por los costes de la mano de obra y la factura energética. Su implantación en el territorio se vio también influida, especialmente después de la "revuelta" de las selfactinas, por la conflictividad laboral, que llevó a muchos empresarios a buscar una mano de obra más dócil fuera de los núcleos urbanos. En definitiva, el industrial catalán no circunscribió su estrategia productiva a un único espacio territorial porque ésta no respondió a un solo condicionante. Al contrario, hubo de adaptarla en función de las circunstancias cambiantes -tanto económicas y tecnológicas, como sociales- a las que debió enfrentarse, apostando por una localización cuando ésta favorecía las posibilidades de negocio o abandonándola cuando ya no respondía adecuadamente a sus necesidades.

\section{Bibliografía}

AGUSTÍ I CULLELL, Jaume (1983): Ciència i tècnica a Catalunya en el segle XVIII o la introducció de la màquina de vapor, Institut d'Estudis Catalans, Barcelona.

AMORÓS, Francesc (1864 [1857]): “Estado demostrativo de las fábricas existentes en el principado de Cataluña (...)", en COLUMBRÍ A., Memorias de un presidario político, Barcelona.

BECATTINI, Giovanni (1979): “Dal settore industriale al distretto industriale. Alcune consideración sull'unità di indagine dell'economia industriale", Rivista di Economia e Politica Industriale, 1, pp. 7-21.

-(2000): Il distretto industriale. Un nuovo modo di interpretare il cambiamento economico, Rosenberg and Séller, Torino.

—(2002): "Del distrito industrial marshalliano a la "teoría del distrito" contemporánea. Una breve reconstrucción crítica", Investigaciones Regionales, 9, pp. 1-32.

BECATTINI, Giovanni; BELLANDI, Marco; DEI OTTATI, Gabi Y SFORZI, Fabio (2003): From Industrial District to Local Development. An Itinerary of Research, E. Elgar, Cheltehnham.

BELLANDI, Marco (2003): "Sistemas productivos locales y bienes públicos específicos", Economiaz, 53, pp. 51-73.

BENAUL, Josep M. ${ }^{a}$ y DEU, Esteve (2005): “L'exploitation industrielle du bassin de Ripoll (XVIII ${ }^{\mathrm{e}}-\mathrm{XX} \mathrm{X}^{\mathrm{e}}$ siècle)", en CAUCANAS, S. y CAZALS, R. (dir.), Du moulin à l'usine. Implantations industrielles, du $X^{e}$ au $X X^{e}$ siècle, Toulouse, Privat, pp. 169-189. 
BENET, Josep Y MARTÍ, Casimir (1976): Barcelona a Mitjan segle XIX. El moviment obrer durant el Bienni Progressista (1854-1856), Barcelona, Curial.

BERG, Maxine (1987): La era de las manufacturas, 1700-1820. Una nueva historia de la revolución industrial británica, Barcelona, Crítica.

BERG, Maxine; HUDSON, Pat y SONENSCHER, Marc (1983) (eds.): Manufacture in Town and Country before the Factory, Cambridge, Cambridge University Press.

BERG, Maxine y BRULAND, Khristine (1998): Technological Revolutions in Europe. Historical Perspectives, Cheltenham, Edward Elgar.

CALVO, Àngel (1999): “La cara conocida. Cambio tecnológico y adaptación al mercado en la industria algodonera catalana (fines s. XIX-comienzos s. XX)", en CARRERAS, A.; PASCUAL, P.; REHER D. et SUDRIÀ C. (dir.), Doctor Jordi Nadal. La industrialització i el desenvolupament econòmic d'Espanya, Barcelona, UB, Vol. II, pp. 1164-1174.

-(2002): "La indústria cotonera a començaments del segle XX", Recerques, 44, pp. 91-110.

CARRERAS, Albert (1983): "El aprovechamiento de la energía hidraúlica en Cataluña, 18401920. Un ensayo de interpretación", Revista de Historia Económica, I (2), pp. 31-63.

CHASSAGNE, Serge (1991): Le coton et ses patrons. France, 1760-1840, Paris, Editions de l'EHESS.

CLUA, Jordi (2001): Procesos hidràulics i aplicació de la legislació pel foment de la població rural a les colònies industrials, Sant Cugat del Vallés, Els llibres de la Frontera.

COHEN, Isaac (1985): "Worker's Control in the Cotton Industry: a Comparative Study of British and American Mule Spinning", Labor History, 26 (1), pp. 53-85.

COOKE, Philip (2002): Knowledge Economies. Clusters, Learning and Cooperative Advantage, Routledge, London.

DELGADO, Josep M. ${ }^{a}$ (1990): “De la filatura manual a la mecànica. Un capítol del desenvolupament de la indústria cotonera a Catalunya (1794-1814)", Recerques, 23, pp. 161-179.

DOMÍNGUEZ, Rafael (2002): La riqueza de las regiones. Las desigualdades económicas regionales en España, 1700-2000, Alianza, Madrid.

DOREL-FERRÉ, Gràcia (1992): Les colònies industrials a Catalunya. El cas de la colònia Sedò, Publicacions de l'Abadia de Montserrat, Barcelona.

—(1999): “L'eau ou le charbon? L'alternative énergétique de l'industrie catalane au XIX cle", en CARRERAS, Albert; PASCUAL P.; REHER D. y SUDRIÀ Carles (dir.), Doctor Jordi Nadal. La industrialització $i$ el desenvolupament econòmic d'Espanya, Barcelona, UB, vol. II, pp. 1057-1067.

-(2004): “Les colonies industrielles, un épisode discuté de l'histoire de la Catalogne des XIX et XX' siècles", en Autour de l'industrie. Histoire et patrimoine, Mélanges offerts à Denis Woronoff, Paris, CHEFF, pp. 265-282.

-(2005): “Le patronat des colonies industrielles catalanes au XIX siècle: formation, diversité, representations", en AUBERT, P.; CHASTAGNARET, G. et RAVEUX, O. (dir.), Construire des mondes. Elites et espaces en Méditerranée, $X V I^{e}-X X^{e}$ siècle, Aix-en-Provence, PUP, pp. 71-85.

ENRECH, Carles (2001): “La ofensiva contra el oficio en la industria textil catalana (18811923). La destrucción de un modelo de sociedad urbana" en CASTILLO, S. y FERNÁNDEZ, R. (coord.), Campesinos, artesanos, trabajadores, Lleida, Editorial Milenio.

-(2003): El pla contra la muntanya. La crisi de la indústria tèxtil del pla i la colonització fabril de la muntanya (1874-1904), Lleida, Edicions de la Universitat de Lleida.

-(2005): Indústria i ofici. Conflicte social i jerarquies obreres a la Catalunya tèxtil (1881-1923), Universitat Autònoma de Barcelona, Barcelona.

FELDMAN, Maryann P. (1994): The Geography of Innovation, Dordrecht, Boston. 
FERRER, LLorenç (1994): “La cintería en Manresa en el siglo XIX. Industiralización y pequeña empresa", en NADAL, Jordi y CATALAN, Jordi (dir.), La cara oculta de la industrialización española. La modernización de los sectores no líderes (s. XIX y XX), Barcelona, Alianza, pp. 225267.

-(2004): “Bergadanas, continuas y mules. Tres geografías de la hilatura del algodón en Cataluña (1790-1830)", Revista de Historia Económica, 22 (2), pp. 337-386.

FERRER, Gerónimo (1846): Cartas históricas, filosóficas, estadísticas, agrícolas, industriales y mercantiles, Barcelona.

FIGUEROLA, Laureano (1849): Estadística de Barcelona en 1849, Madrid, Instituto de Estudios Fiscales, 1968.

GARCÍA BALAÑ̇̀, Albert (2002): “Indústria i ordre social: una lectura política del treball cotoner a la Barcelona del segle XIX", en FRADERA, J. M. (dir.), Societat, política i cultura a Catalunya, 1830-1880, Barcelona, Ajuntament de Barcelona, Quaderns d'Història, 6, pp. 51-73.

-(2004): La fabricació de la fabrica. Treball i politica a la Catalunya cotonera (1784-1874), Barcelona, Publicacions de l'Abadia de Montserrat.

GERMÁN, Luis; LLOPIS, Enrique; MALUQUER DE MOTES, Jordi y ZAPATA, Santiago (eds.) (2001): Historia económica regional de España (siglos XIX y XX), Barcelona, Ariel.

GRAELL, Guillem (1911): Historia del Fomento del Trabajo Nacional, Barcelona.

GUTIÉRREZ, Manuel M. ${ }^{a}$ (1837): Impugnación a las cinco proposiciones de Pebrer sobre los grandes males que causa la ley de aranceles a la nación en general, a la Cataluña en particular, y a las mismas fábricas catalanas, Madrid.

GUTIÉRREZ, M. ${ }^{a}$ LLuisa (1997): La España Industrial, 1847-1853. Un model d'innovació tecnològica, Barcelona, Associació/Col.legi d'Enginyers Industrials de Catalunya.

HARRIS, John R. (1998): Industrial Espionage and Technology Transfer: Britain and France in the Eighteenth Century, Aldershot.

HUDSON, Pat (1989) (ed.): Regions and Industries: a Perspective on the Industrial Revolution in Britain, Cambridge, CUP.

LAZONICK, William (1991): Competitive Advantage on the Sho Floor, Cambridge, Havard University Press.

LESCURE, Michel (2002): "Le territoire, comme organisation et comme institution", en La mobilisation du territoire. Les districtes industriels en Europe occidentale du XVIIe au XXe siècles, Paris, Comité pour 1'Histoire Economique et Financière de la France.

MADOZ, Pascual (1846): Diccionario geográfico-estadístico-histórico de España y sus posesiones de Ultramar, Madrid, Vol. III.

MALUQUER DE MOTES, Jordi (1976): “La estructura del sector algodonero en Cataluña durante la primera etapa de la industrialización (1832-1861)", Hacienda Pública Española, 38, pp. 133-148.

—(1990): “Las técnicas hidráulicas y la gestión del agua en la especialización industrial de Cataluña. Su evolución a largo plazo", en PÉREZ PICAZO, M. T. y LEMEUNIER, G. (eds.), Agua y modo de producción, Barcelona, Editorial Crítica, pp. 311-348.

-(2000) (dir.): Tècnics i tecnología en el desenvolupament de la Catalunya contemporània, Barcelona, Enciclopèdia Catalana.

MIRANDA, José Antonio (2004): “Industrialización y distritos industriales: la Comunidad Valenciana", en DI VITTORIO, A.; BARCIELA, C. y FONTANA, G.L. (eds.), Storiografia d'industria e d'impresa in Italia e Spagna in età moderna e contemporanea, Padova, CLEUP.

-(2005): “Calzados y distritos industriales en el Mediterráneo", Mediterráneo Económico, 7, pp. 289-312. 
NADAL, Jordi (1975): El fracaso de la Revolución industrial en España, 1814-1913, Barcelona, Ariel.

-(1985): "La formación de la industria moderna", en Catalunya, la fabrica d'Espanya. Un siglo de industrialización catalana, 1833-1936, Barcelona, Generalitat de Catalunya, pp. 43-113.

-(1991): "La indústria cotonera" en Història econòmica de la Catalunya contemporània, Barcelona, Enciclopèdia Catalana, III, pp. 12-85.

-(1992): “Los Planas, constructores de turbinas y material eléctrico", Revista de Historia Industrial, 1, pp. 63-94.

-(2000): “Somni i realitat en la primera industrialització catalana. L'intent, fallit, d'industrialització a l'anglesa", en El segle romàntic. Actes del col.loqui sobre Josep Yxart i el seu temps. Tarragona, 23, 24 i 25 de novembre de 1995, Tarragona, Diputació de Tarragona, pp. 529-540.

NADAL, Jordi y CARRERAS, Albert (1990) (coords.): Pautas regionales de la industrialización española. Siglos XIX y XX, Barcelona, Ariel.

NADAL, Jordi y TAFUNELL, Xavier (1992): Sant Martí de Provençals, pulmó industrial de Barcelona (1847-1992), Barcelona, Columna.

OKUNO, Yositomo (1999): “Entre la llana i el cotó. Una nota sobre l'extensió de la indústria del cotó als pobles de Catalunya en el darrer quart del segle XVIII", Recerques, 38, pp. 47-76.

OLIVA, Bernat (1999): Els orígens de la primera industriatlizació del rerepaís. Un cas emblemàtic : Vilasar i el capital comercial barceloní (1828-1875), Mataró, Caixa d'Estalvis Laietana.

-(2000): “Capital comercial i primera industrialització. La casa de comerç Nadal y Ribó i la industrialització catalana a mitjan segle XIX", Estudis Històrics i Documents dels Arxius de Protocols, 18, pp. 373-417.

PALUZIE, Elisenda; PONS, Jordi y TIRADO, Daniel (2002): “The Geographical Concentration of Industry Accross Spanish Regions, 1856-1995", Documents de Treball de la Divisió de Ciències Jurídiques, Econòmiques $i$ Socials, n. ${ }^{\circ}$ E02/86, Universitat de Barcelona.

PAREJO, Antonio (2006): “De la región a la ciudad. Hacia un nuevo enfoque de la historia industrial española contemporánea", Revista de Historia Industrial, 30, pp. 53-102.

PASCUAL, Pere (1991): “La Diputació i la modernització de la xarxa viària catalana: 18401868", en DE RIQUER, Borja (ed.), Història de la Diputació de Barcelona, Barcelona, Diputació de Barcelona, pp. 142-193.

-(1999): Los caminos de la era industrial. La construcción y financiación de la Red Ferroviaria Catalana (1843-1898), Barcelona, PUB.

POLLARD, Sidney (1981): Peaceful Conquest. The Industrialization of Europe, 1760-1970. Oxford, OUP, (traducción castellana de 1991).

-(1994): "Regional and Inter-regional Economic Development in Europe in the Eighteenth and Nineteenth Centuries", Debates and Controversies in Economic History. A-sessions. Proccedings Eleventh International Economic History Congress, Milan, pp. 57-94.

RAVEUX, Olivier (2005): “Los fabricantes de algodón de Barcelona (1833-1844): estrategias empresariales en la modernización de un distrito industrial", Revista de Historia Industrial, 28, pp. 157-185.

REDDY, William (1984): The Rise of Market Culture. The Textile Trade and French Society, 17501900, Cambridge/Paris, Cambridge University Press /Editions de la Maison des Sciences de l'Homme.

ROSÉS, Joan Ramon (2001a): “La competitividad internacional de la industria algodonera española (1830-1860)", Revista de Historia Económica, 19, pp. 85-108. 
—(2001b): “La difusión de la tecnología extranjera en España durante la Revolución Industrial: el caso de la industria algodonera catalana (1784-1861)", en VII Congreso de la Asociación de Historia Económica. Zaragoza (http:/ / www.unizar.es/ eueez/cahe/ roses.pdf.).

-(2003): "Why isn't the Whole of Spain Industrialized? New Economic Geography and Early Industrialization (1797-1910)", Journal of Economic History, Vol. 63, 4, pp. 995-1022.

SÁNCHEZ, Alex (1987): “Los inicios del asociacionismo empresarial en España: la Real Compañía de Hilados de Algodón de Barcelona, 1772-1820", Hacienda Pública Española, $108 / 109$, pp. 253-268.

-(1989): “Entre el tradicionalismo manufacturero y la modernización industrial. El cuerpo de Fabricantes de Tejidos e Hilados de Algodón de Barcelona, 1799-1819", Estudis d'Història Econòmica, 1, pp. 71-88.

-(1996): "La empresa algodonera en Cataluña antes de la aplicación del vapor, 1783-1832", en COMÍN, Francisco y MARTÍN ACEÑA, Paco (eds.), La empresa en la historia de España, Madrid, Civitas, pp. 155-170.

—(2000a): “Crisis económica y respuesta empresarial. Los inicios del sistema fabril en la industria algodonera catalana, 1797-1839", Revista de Historia Económica, 18 (3), pp. 485523.

—(2000b): "Les berguedanes i les primeres màquines de filar", en MALUQUER DE MOTES, Jordi (dir.), Tècnics i tecnologia en el desenvolupament de la Catalunya contemporània, Barcelona, Enciclopèdia Catalana, pp. 161-175.

SARD, Andrés de (1884): Comparación entre el actual estado de desarrollo de la industria algodonera en Inglaterra y el de la propia industria en España, Barcelona.

SMITH, Angel (2003): "Industria, oficio y género en la industria textil catalana, 1833-1923", Historia Social, 45, pp. 79-99.

SOLÀ, Àngels (1995): “Indústria tèxtil, màquines i fàbriques a Berga", l'Erol, 47, pp. 12-15.

—(2002): "Filar amb berguedanes. Mite i realitat d'una màquina de filar cotó", en La Indùstria textil. Actes de les V Jornades d'Arqueologia Industrial de Catalunya, Barcelona, Enginyers Industrials de Catalunya, pp. 143-168.

-(2004a): Aigua, indústria i fabricants a Manresa (1759-1860), Manresa, Centre d'Estudis del Bages.

-(2004b): "Els Herp de Manresa, una saga de negociants i fabricants en els orígens de la Catalunya industrial (1800-1859)", Estudis Històrics i Documents dels Arxius de Protocols, 22, pp. 251- 311.

SOLER, Raimon (2001a): "Estratègies empresarials en la indústria cotonera catalana. El cas de la Fàbrica de la Rambla de Vilanova, 1833-1965", Tesis Doctoral Inédita, UB.

-(2001b): "Productividad, costes y eficiencia en la industria textil algodonera catalana, 18401930 ¿Qué nos enseñan las empresas?", en VII Congreso de la Asociación de Historia Económica. Zaragoza (http:/ / www.unizar.es/eueez/cahe/ raimonsoler.pdf).

TERRADAS Ignasi (1994): La qüestió de les colònies industrials. L'exemple de l'Ametlla de Merola, Manresa, Centre d'Estudis del Bages.

THOMSON, James (1994): Els orígens de la industrialització a Catalunya. El cotó a Barcelona, 17281832, Barcelona, Edicions, 62.

-(2003a): "Transferencia tecnológica en la industria algodonera catalana: de las indianas a la selfactina", Revista de Historia Industrial, 24, pp. 13-50.

-(2003b): "Olot, Barcelona and Avila and the Introduction of the Arkwright Technology to Catalonia", Revista de Historia Económica, XXI, 2, pp. 297-334.

-(2005): "Explaining the <take-off $>$ of the Catalan Cotton Industry", Economic History Review, LVIII, 4, pp. 701-735. 
TIRADO, Daniel; PONS, Jordi y PALUZIE, Elisenda (2003): “Industrial Agglomeration and Industrial Location. The Case of Spain before World War I", Journal of Economic Geography, 2, pp. 343-363.

-(2006): "Los cambios en la localización de la actividad industrial en España, 1850-1936", Revista de Historia Industrial, 31, pp. 41-63.

TORRAS, Jaume (1994): “L'economia catalana abans del 1800. Un esquema”, en Història econòmica de la Catalunya contemporània, Barcelona, Enciclopèdia Catalana, I, pp. 13-38.

TUNZELMAN, Georg N. von (1978): Steam Power and British Industrialization to 1860, Oxford, OUP. 